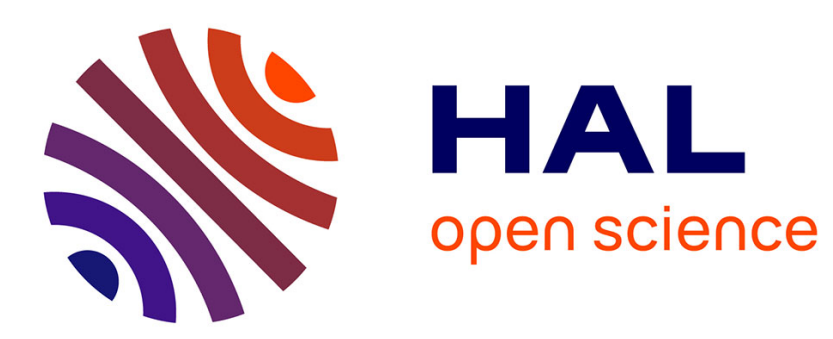

\title{
Small-inclusion asymptotic of misfit functionals for inverse problems in acoustics
}

\author{
B. B. Guzina, Marc Bonnet
}

\section{To cite this version:}

B. B. Guzina, Marc Bonnet. Small-inclusion asymptotic of misfit functionals for inverse problems in acoustics. Inverse Problems, 2006, 22, pp.1761-1785. 10.1088/0266-5611/22/5/014 . hal-00111266

\section{HAL Id: hal-00111266 \\ https://hal.science/hal-00111266}

Submitted on 9 Aug 2008

HAL is a multi-disciplinary open access archive for the deposit and dissemination of scientific research documents, whether they are published or not. The documents may come from teaching and research institutions in France or abroad, or from public or private research centers.
L'archive ouverte pluridisciplinaire HAL, est destinée au dépôt et à la diffusion de documents scientifiques de niveau recherche, publiés ou non, émanant des établissements d'enseignement et de recherche français ou étrangers, des laboratoires publics ou privés. 


\title{
Small-inclusion asymptotic of misfit functionals for inverse problems in acoustics
}

\author{
Bojan B. Guzina† and Marc Bonnet \\ $\dagger$ Civil Engineering, University of Minnesota, Minneapolis, USA \\ E-mail: guzina@wave.ce.umn.edu \\ $\ddagger$ Laboratoire de Mécanique des Solides, Ecole Polytechnique, Palaiseau, France \\ E-mail: bonnet@lms.polytechnique.fr
}

\begin{abstract}
.
The aim of this study is an extension and employment of the concept of topological derivative as it pertains to the nucleation of infinitesimal inclusions in a reference (i.e. background) acoustic medium. The developments are motivated by the need to develop a preliminary indicator functional that would aid the solution of inverse scattering problems in terms of a rational initial "guess" about the geometry and material characteristics of a hidden (finite) obstacle; an information that is often required by iterative minimization algorithms. To this end the customary definition of topological derivative, that quantifies the sensitivity of a given cost functional with respect to the creation of an infinitesimal hole, is adapted to permit the nucleation of a dissimilar acoustic medium. On employing the Green's function for the background domain, computation of topological sensitivity for the three-dimensional Helmholtz equation is reduced to the solution of a reference, Laplace transmission problem. Explicit formulas are given for the nucleating inclusions of spherical and ellipsoidal shape. For generality the developments are also presented in an alternative, adjoint-field setting that permits nucleation of inclusions in an infinite, semi-infinite or finite background medium. Through numerical examples it is shown that the featured topological sensitivity could be used, in the context of inverse scattering, as an effective obstacle indicator through an assembly of sampling points where it attains pronounced negative values. On varying a material characteristic (density) of the nucleating obstacle, it is also shown that the proposed methodology can be used as a preparatory tool for both geometric and material identification.
\end{abstract}

Keywords: Topological derivative, Helmholtz equation, inverse scattering, transmission problem, acoustic waves, penetrable obstacles, probe method.

\section{Introduction}

Shape reconstruction of obstacles embedded in an acoustic medium, using either faror near-field patterns of the scattered wave field, is a challenging subject $[12,10]$ with application to diverse areas such as sonar detection and medical imaging. In view of their non-linear nature, inverse problems of this class are often dealt with by way of gradient-based optimization [13, 15], sometimes aided by the adjoint-field sensitivity

† Inverse Problems 22:1761-1785 (2006) 
estimates [5] for computational efficiency and level-set methodology [24] as a means to permit changes in topology of the trial scatterer. Unfortunately, cost functionals associated with shape reconstruction problems are often non-convex [26] which renders the success of gradient-based optimization highly dependent on the initial "guess" in terms of the location, topology, and geometry of the scatterer. In the absence of suitable prior information, a direct way to deal with the problem revolves around the use of global search algorithms $[25,33]$. Owing to their exceeding computational cost, however, such solutions are by rule impractical which exposes the need for computationally effective, preliminary global-search tools whose primary purpose would be to generate a reliable initial "guess" for gradient-based optimization.

Building on the results in shape optimization obtained for Laplace [32, 19] and Helmholtz [30, 27] systems, [20, 8, 18] and [17] have recently established the method of topological sensitivity as a tool for preliminary, grid-based reconstruction of obstacles in the context of inverse elastic and acoustic scattering. In the approach the topological derivative, which quantifies the sensitivity of a given cost functional with respect to the nucleation of an infinitesimal obstacle in the reference (background) medium, is used as a spatial obstacle indicator. Notwithstanding their usefulness, however, the foregoing topological sensitivity analyses are limited in the sense that they are focused on the nucleation of impenetrable scatterers with either Dirichlet or Neumann conditions imposed on their boundary. To deal with elastic-wave identification of penetrable obstacles, in terms of their both geometric and material features, [21] have recently developed a generalization of topological sensitivity that postulates the nucleation of dissimilar elastic inclusions. In this study, the latter idea is carried out to deal with inverse scattering problems in acoustics. In particular, the proposed "material-topological" sensitivity is shown to consist of a monopole term, related to the compressibility contrast, and a dipole term involving the mass density contrast. For generality, the proposed developments are also cast within the adjoint-field formulation, an alternative framework allowing efficient computation of the material-topological sensitivity in an arbitrary (infinite or finite, homogeneous or heterogeneous) background acoustic medium. Through numerical examples it is shown that the material-topological sensitivity can be used, in the context of inverse scattering, as an effective obstacle indicator through an assembly of sampling points where it attains pronounced negative values. On varying the material characteristics of a nucleating obstacle, the proposed indicator is also shown to permit both preliminary geometric and material identification. The latter result may be for instance useful in breast cancer detection wherein the mechanical characteristics of a lesion, detected e.g. via ultrasound or magnetic resonance imaging, may allow one to differentiate between malignant and benign growths $[31,16]$.

For generality, it is noted that the idea of (preliminary) obstacle reconstruction via spatial sampling of a given indicator functional is shared by a diverse array of inverse scattering techniques, now commonly referred to as the "sampling" or "probe" methods [28], which notably include the linear sampling method, e.g. [11, 10]. In this context, an interesting observation is that the linear sampling and topological 
sensitivity methods both revolve around the evaluation of a probing functional that projects the relevant (background) Green's function onto a suitable function space synthesizing the experimental observations. However, these two methods have distinct theoretical frameworks, and their relationship is very much an open research issue. As one of the key distinctions relevant to this study, the derivation of materialtopological sensitivity formulae for a given cost functional requires an asymptotic expansion of the scattered field induced by the nucleating penetrable obstacle. Although the related asymptotic expressions have been proposed elsewhere (e.g. [2]) for inverse electromagnetic problems and penetrable scatterers, they have so far been utilized for the explicit reconstruction of small inhomogeneities based on either i) treatment of the measurement residuals $[3,34,35]$, or ii) the reciprocity-gap approach [4]. In contrast, the present approach, that makes use of the spatial distribution of topological sensitivity as an indicator functional, aims at preliminary reconstruction of finite obstacles and may broadly be categorized as a sampling technique exploiting asymptotic expansions.

\section{Preliminaries}

With reference to the Cartesian frame $\left\{O ; \xi_{1}, \xi_{2}, \xi_{3}\right\}$, consider a semi-infinite acoustic domain $\Omega=\left\{\boldsymbol{\xi} \mid \xi_{3}<0\right\}$ housing a penetrable obstacle $B$ that is bounded by the closed, piecewise-smooth surface $S$ (Fig. 1). The reference background medium and the obstacle are each assumed to be homogeneous with wave speed and mass density $(c, \rho)$ and $\left(c^{*}, \rho^{*}\right)$, respectively. In this setting, $\Omega^{-}=\Omega \backslash(B \cup S)$ is the exterior region surrounding the obstacle, and $\bar{\Omega}^{-}=\Omega^{-} \cup \Sigma \cup S$ denotes the closure of $\Omega^{-}$where $\Sigma=\left\{\boldsymbol{\xi} \mid \xi_{3}=0\right\}$ is the "top" surface of the half-space. For further reference the normal on $\Sigma \cup S$, oriented outward from $\Omega^{-}$, will be denoted by $\boldsymbol{n}$. The homogeneous boundary conditions on $\Sigma$ are assumed to be either of the Dirichlet or Neumann type. With the implicit timeharmonic factor $e^{i \omega t}$ omitted henceforth for brevity, let the obstacle be illuminated by the

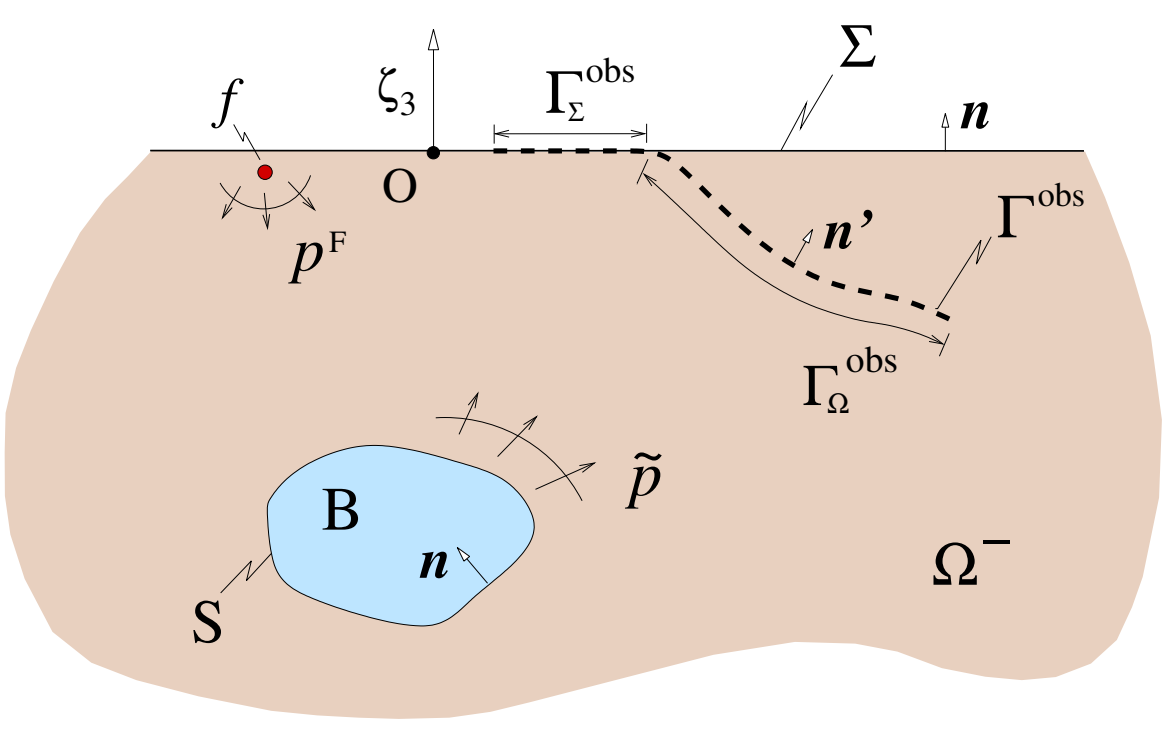

Figure 1. Configuration of the scattering problem 
free (i.e. incident) acoustic field $p^{\mathrm{F}}$ defined as the response of an obstacle-free half-space due to prescribed source distribution $f_{\Omega}(\boldsymbol{\xi})$ and boundary excitation $f_{\Sigma}(\boldsymbol{\xi})$ so that

$$
\begin{array}{ll}
\nabla^{2} p^{\mathrm{F}}+k^{2} p^{\mathrm{F}}+f_{\Omega}=0, & \boldsymbol{\xi} \in \Omega, \\
\alpha p^{\mathrm{F}}+(1-\alpha) p_{, n}^{\mathrm{F}}=f_{\Sigma}, & \boldsymbol{\xi} \in \Sigma .
\end{array}
$$

Here $k=\omega / c ; g_{, n}=\boldsymbol{n} \cdot \nabla g$, and $\alpha$ takes the respective values 0 and 1 when $\Sigma$ is the Neumann and Dirichlet boundary. In what follows, it is assumed that $\omega$ is not a fictitious eigenfrequency of any of the featured boundary-value problems.

When an obstacle is present, the prescribed excitation $f \equiv\left(f_{\Omega}, f_{\Sigma}\right)$ gives rise to the acoustic field $p$ in $\Omega^{-} \cup B$ that can be conveniently decomposed as

$$
p(\boldsymbol{\xi})=p^{\mathrm{F}}(\boldsymbol{\xi})+\tilde{p}(\boldsymbol{\xi}), \quad \boldsymbol{\xi} \in \Omega^{-}
$$

in the exterior domain where $\tilde{p}$ denotes the perturbation component, termed the scattered field. Assuming that the free field $p^{\mathrm{F}}$ is known beforehand, the forward scattering problem, i.e. the task of calculating $p$ in $\Omega^{-} \cup B$ (given $f$ ) can be cast in terms of $\left.\tilde{p}\right|_{\Omega^{-}}$and $\left.p\right|_{B}$ as

$$
\begin{gathered}
\nabla^{2} \tilde{p}+k^{2} \tilde{p}=0, \quad \boldsymbol{\xi} \in \Omega^{-}, \\
\nabla^{2} p+\gamma^{2} k^{2} p=0, \quad \boldsymbol{\xi} \in B, \\
\alpha \tilde{p}+(1-\alpha) \tilde{p}_{, n}=0, \quad \boldsymbol{\xi} \in \Sigma \\
\tilde{p}+p^{\mathrm{F}}=p, \quad \tilde{p}_{, n}+p_{, n}^{\mathrm{F}}=\beta p_{, n} \quad \boldsymbol{\xi} \in S,
\end{gathered}
$$

where $\beta=\rho / \rho^{*}, \gamma=c / c^{*}$, and the normal derivatives $\tilde{p}_{, n}, p_{, n}^{\mathrm{F}}$ are defined in terms of the same normal $\boldsymbol{n}$. To ensure physical relevance, the scattered field is further assumed to satisfy the regularity and radiation conditions

$$
\tilde{p}=O\left(\frac{1}{R}\right), \quad \tilde{p}_{\mathrm{R}}+i k \tilde{p}=o\left(\frac{1}{R}\right), \quad R \equiv|\boldsymbol{\xi}| \rightarrow \infty, \quad \boldsymbol{\xi} \in \Omega^{-} .
$$

For identification purposes, let $\Gamma^{\text {obs }} \subset \Sigma \cup \Omega^{-}$denote the measurement surface. With the foregoing definitions, the inverse problem of interest can be stated as a task of resolving the "true" obstacle $B^{\text {true }}$, both in terms of its geometry and material characteristics $\left(c^{* \text {,true }}, \rho^{* \text {,true }}\right)$, from the knowledge of: i) time-harmonic source $f$ used to illuminate the obstacle, ii) acoustic properties $(c, \rho)$ of the background medium, and iii) distribution of the induced pressure field, $p^{\text {true }}$, as observed over the measurement surface $\Gamma^{\text {obs }}$. In what follows, these measurements will be denoted by $p^{\text {obs }}$, so that $p^{\text {obs }}(\boldsymbol{\xi})=p^{\text {true }}(\boldsymbol{\xi}), \quad \boldsymbol{\xi} \in \Gamma^{\text {obs }}$ under ideal modeling and measurement conditions. For generality, it is assumed that either $\Gamma^{\text {obs }} \cap \Sigma \equiv \Gamma_{\Sigma}^{\text {obs }}$ or $\Gamma^{\text {obs }} \cap \Omega^{-} \equiv \Gamma_{\Omega}^{\text {obs }}$ (see Fig. 1) may be an empty set.

For a systematic treatment of the identification problem, a cost functional is established that quantifies the misfit between experimental observations $p^{\text {obs }}$ and their acoustic predictions $p$, calculated for a trial obstacle $B$. To provide a focus in the study, the ensuing discussion deals with the class of cost functionals

$$
\mathcal{J}\left(\Omega^{-}, \beta, \gamma ; f\right)=\int_{\Gamma_{\mathrm{obs}}} \varphi(p(\boldsymbol{\xi}), \boldsymbol{\xi}) \mathrm{d} \Gamma_{\xi}
$$


where $\beta$ and $\gamma$ synthesize the material characteristics of a trial obstacle, while the distance function $\varphi$ is assumed to be real-valued, nonnegative, and differentiable with respect to the real and imaginary parts of its first argument. In waveform tomography, $\varphi$ commonly takes the weighted least-squares format

$$
\varphi(p(\boldsymbol{\xi}), \boldsymbol{\xi})=\frac{1}{2} W(\boldsymbol{\xi}) \overline{\left\{p(\boldsymbol{\xi})-p^{\mathrm{obs}}(\boldsymbol{\xi})\right\}}\left\{p(\boldsymbol{\xi})-p^{\mathrm{obs}}(\boldsymbol{\xi})\right\}, \quad W(\boldsymbol{\xi})>0 .
$$

\subsection{Green's functions}

With an implicit time-harmonic factor $e^{i \omega t}$ as examined earlier, let

$$
G(\boldsymbol{\xi}, \boldsymbol{x} ; k)=\frac{e^{-i k r}}{4 \pi r}, \quad H(\boldsymbol{\xi}, \boldsymbol{x} ; k) \equiv \boldsymbol{n} \cdot \nabla G(\boldsymbol{\xi}, \boldsymbol{x} ; k)=-\frac{e^{-i k r}}{4 \pi r^{2}}(1+i k r) r_{, n},
$$

where $r=|\boldsymbol{\xi}-\boldsymbol{x}|$ and $\nabla G$ signifies the gradient of $G$ with respect to its first argument, denote the acoustic fundamental solution for the free space with wavenumber $k$, so that

$$
\nabla^{2} G(\boldsymbol{\xi}, \boldsymbol{x} ; k)+k^{2} G(\boldsymbol{\xi}, \boldsymbol{x} ; k)+\delta(\boldsymbol{\xi}-\boldsymbol{x})=0, \quad \boldsymbol{\xi} \in \mathbb{R}^{3} .
$$

On the basis of (7), the Green's function for the reference (i.e. obstacle-free) half-space $\Omega$ can be written as

$$
\begin{aligned}
& \hat{G}(\boldsymbol{\xi}, \boldsymbol{x} ; k)=G(\boldsymbol{\xi}, \boldsymbol{x} ; k)+(1-2 \alpha) G\left(\boldsymbol{\xi}, \boldsymbol{x}^{\prime} ; k\right), \\
& \hat{H}(\boldsymbol{\xi}, \boldsymbol{x} ; k)=H(\boldsymbol{\xi}, \boldsymbol{x} ; k)+(2 \alpha-1) H\left(\boldsymbol{\xi}, \boldsymbol{x}^{\prime} ; k\right),
\end{aligned}
$$

where $\boldsymbol{x}$ and $\boldsymbol{x}^{\prime}$ are symmetric with respect to the top surface of the half-space, and $\alpha \in\{0,1\}$ follows the convention introduced in (1).

By virtue of the foregoing definitions, the free field satisfying (1) can be written as

$$
p^{\mathrm{F}}(\boldsymbol{x})=\int_{\Omega^{-}} f_{\Omega}(\boldsymbol{\xi}) \hat{G}(\boldsymbol{\xi}, \boldsymbol{x}, k) d V_{\xi}+\int_{\Sigma} f_{\Sigma}(\boldsymbol{\xi})[\alpha \hat{H}(\boldsymbol{\xi}, \boldsymbol{x}, k)+(1-\alpha) \hat{G}(\boldsymbol{\xi}, \boldsymbol{x}, k)] \mathrm{d} \Gamma_{\xi} .
$$

\section{Generalized Topological Sensitivity}

This section is focused on the development of topological derivative for the class of cost functionals $\mathcal{J}$ given by (5). To this end, let $\mathscr{B} \subset \mathbb{R}^{3}$ be a fixed bounded open set with volume $|\mathscr{B}|$ containing the origin. For generality, it is assumed that $\mathscr{S}=\partial \mathscr{B}$ is piecewisesmooth with components of class $C^{2}$. With such definitions, one may consider the introduction of a small penetrable obstacle $B_{\epsilon}\left(\boldsymbol{x}^{\circ}\right)=\boldsymbol{x}^{\circ}+\epsilon \mathscr{B}$ with material properties $c^{*}=c / \gamma, \rho^{*}=\rho / \beta$ and size $\epsilon>0$, at a fixed trial point $\boldsymbol{x}^{\circ}$ in an otherwise obstacle-free half-space $\Omega$ with properties $c$ and $\rho$. Following [32, 9], one is in particular interested in the asymptotic behavior of $\mathcal{J}\left(\Omega_{\epsilon}^{-}, \beta, \gamma ; f\right)$ for infinitesimal $\epsilon>0$, where $\Omega_{\epsilon}^{-}=\Omega \backslash \bar{B}_{\epsilon}\left(\boldsymbol{x}^{\circ}\right)$ and $\bar{B}_{\epsilon}=B_{\epsilon} \cup S_{\epsilon}$ is the closure of $B_{\epsilon}\left(\boldsymbol{x}^{\circ}\right) \subset \Omega$. With reference to this limiting behavior, the topological derivative $\mathcal{T}\left(\boldsymbol{x}^{\circ}, \beta, \gamma ; f\right)$ of the cost functional (5) for an obstacle-free body can be defined through the expansion

$$
\mathcal{J}\left(\Omega_{\epsilon}^{-}, \beta, \gamma ; f\right)=\mathcal{J}(\Omega ; f)+\mathcal{T}\left(\boldsymbol{x}^{\circ}, \beta, \gamma ; f\right) h(\epsilon)+o(h(\epsilon)), \quad \epsilon \rightarrow 0
$$


where $\mathcal{J}(\Omega ; f) \equiv \mathcal{J}(\Omega, \beta, \gamma ; f)$ denotes the degenerate value of (5) when $p=p^{\mathrm{F}}$ and $h(\epsilon)>0$, characterizing the leading term, is to be determined. For the concept of topological derivative to make sense, it is assumed that

$$
\lim _{\epsilon \rightarrow 0} h(\epsilon)=0, \quad\left|\mathcal{T}\left(\boldsymbol{x}^{\circ}, \beta, \gamma ; f\right)\right|<\infty, \quad \boldsymbol{x}^{\circ} \in \Omega .
$$

In general, the functional form of $h(\epsilon)$ depends on the nature of the governing field equation, the type of boundary conditions prescribed on $\mathscr{S}$, and dimensionality of the problem $[9,19]$. As examined earlier, the focus of this study is the expansion (11), and in particular its leading-term coefficient $\mathcal{T}$, for the three-dimensional Helmholtz equation in the context of cost functionals (5) and a penetrable obstacle problem.

With reference to $(2)$ and $(5)$, evaluation of $\mathcal{J}\left(\Omega_{\epsilon}^{-}, \beta, \gamma ; f\right)$ requires the knowledge of the acoustic solution, $\tilde{p}=\tilde{p}^{\epsilon}$, to the forward transmission problem (3) wherein $B$ is replaced by $B_{\epsilon} \equiv B_{\epsilon}\left(\boldsymbol{x}^{\circ}\right)$. Since the scattered field $\tilde{p}^{\epsilon}$ is expected to vanish as $\epsilon \rightarrow 0$ whereas the free field $p^{\mathrm{F}}$ does not depend on $\epsilon$, the leading contribution to $\mathcal{J}\left(\Omega_{\epsilon}^{-}, \beta, \gamma ; f\right)$ can be sought on the basis of an expansion with respect to $\tilde{p}^{\epsilon}$, i.e.

$$
\mathcal{J}\left(\Omega_{\epsilon}^{-}, \beta, \gamma ; f\right)=\mathcal{J}(\Omega ; f)+\int_{\Gamma^{\mathrm{obs}}} \operatorname{Re}\left\{\frac{\partial \varphi}{\partial p}\left(p^{\mathrm{F}}(\boldsymbol{\xi}), \boldsymbol{\xi}\right) \tilde{p}^{\epsilon}(\boldsymbol{\xi})\right\} \mathrm{d} \Gamma_{\xi}+O\left(\left\|\tilde{p}^{\epsilon}\right\|^{2}\right),
$$

where

$$
\frac{\partial \varphi}{\partial p} \equiv \frac{\partial \varphi}{\partial p_{R}}-i \frac{\partial \varphi}{\partial p_{I}} \quad\left(p_{R}=\operatorname{Re}(p), p_{I}=\operatorname{Im}(p)\right)
$$

By means of (11) and (13), the topological derivative of $\mathcal{J}$ can be recast as

$$
\mathcal{T}\left(\boldsymbol{x}^{\circ}, \beta, \gamma ; f\right)=\lim _{\epsilon \rightarrow 0} \frac{1}{h(\epsilon)} \int_{\Gamma^{\mathrm{obs}}} \operatorname{Re}\left\{\frac{\partial \varphi}{\partial p}\left(p^{\mathrm{F}}(\boldsymbol{\xi}), \boldsymbol{\xi}\right) \tilde{p}^{\epsilon}(\boldsymbol{\xi})\right\} \mathrm{d} \Gamma_{\xi} .
$$

The direct approach for the computation of topological derivative proposed in this study represents a generalization of the methodology developed in [20] for the inverse scattering problems in infinite and semi-infinite media. It entails a substitution of the leading contribution of $\tilde{p}^{\epsilon}$ into (14) and seeking the limit of the resulting expression as $\epsilon \rightarrow 0$. To this end, it is useful to recall the integral representation

$$
\tilde{p}^{\epsilon}(\boldsymbol{x})=\int_{S_{\epsilon}} \tilde{p}_{, n}^{\epsilon}(\boldsymbol{\xi}) \hat{G}(\boldsymbol{\xi}, \boldsymbol{x}, k) d S_{\xi}-\int_{S_{\epsilon}} \tilde{p}^{\epsilon}(\boldsymbol{\xi}) \hat{H}(\boldsymbol{\xi}, \boldsymbol{x}, k) d S_{\xi}, \quad \boldsymbol{x} \in \Omega_{\epsilon}^{-}
$$

of the scattered field induced by $B_{\epsilon}$. On employing the interfacial conditions over $S=S_{\epsilon}$ as in (3), the divergence theorem, and the Taylor expansion of the featured Green's function at $\boldsymbol{\xi}=\boldsymbol{x}^{\circ}$, the limiting behavior of (15) for a vanishing obstacle can be written as

$$
\begin{aligned}
\tilde{p}^{\epsilon}(\boldsymbol{x})=( & 1-\beta)\left\{\int_{B_{\epsilon}} \nabla p^{\epsilon}(\boldsymbol{\xi}) d V_{\xi}\right\} \cdot \nabla \hat{G}\left(\boldsymbol{x}^{\circ}, \boldsymbol{x}, k\right) \\
& -\left(1-\beta \gamma^{2}\right) k^{2}\left\{\int_{B_{\epsilon}} p^{\epsilon}(\boldsymbol{\xi}) d V_{\xi}\right\} \hat{G}\left(\boldsymbol{x}^{\circ}, \boldsymbol{x}, k\right)+o\left(\epsilon^{3}\right), \quad \boldsymbol{x} \in \Omega_{\epsilon}^{-}, \quad \epsilon \rightarrow 0
\end{aligned}
$$

One may note that (16) requires the knowledge of $p^{\epsilon}$ and $\nabla p^{\epsilon}$ in the interior of a vanishing obstacle, quantities that are unknown beforehand. Their evaluation is addressed next. 


\subsection{Small-inclusion asymptotics}

The forward problem (3) for $B=B_{\epsilon}$ can be conveniently formulated in terms of a pair of boundary integral equations

$$
\left.\begin{array}{l}
\kappa(\boldsymbol{x}) \tilde{p}^{\epsilon}(\boldsymbol{x})+\int_{S_{\epsilon}} \tilde{p}^{\epsilon}(\boldsymbol{\xi}) \hat{H}(\boldsymbol{\xi}, \boldsymbol{x}, k) d S_{\xi}-\int_{S_{\epsilon}} \tilde{p}_{, n}^{\epsilon}(\boldsymbol{\xi}) \hat{G}(\boldsymbol{\xi}, \boldsymbol{x}, k) d S_{\xi}=0, \\
\kappa(\boldsymbol{x}) \tilde{p}^{\epsilon}(\boldsymbol{x})-\int_{S_{\epsilon}} \tilde{p}^{\epsilon}(\boldsymbol{\xi}) H(\boldsymbol{\xi}, \boldsymbol{x}, \gamma k) d S_{\xi}+\frac{1}{\beta} \int_{S_{\epsilon}} \tilde{p}_{, n}^{\epsilon}(\boldsymbol{\xi}) G(\boldsymbol{\xi}, \boldsymbol{x}, \gamma k) d S_{\xi}= \\
-\kappa(\boldsymbol{x}) p^{\mathrm{F}}(\boldsymbol{x})+\int_{S_{\epsilon}} p^{\mathrm{F}}(\boldsymbol{\xi}) H(\boldsymbol{\xi}, \boldsymbol{x}, \gamma k) d S_{\xi}-\frac{1}{\beta} \int_{S_{\epsilon}} p_{, n}^{\mathrm{F}}(\boldsymbol{\xi}) G(\boldsymbol{\xi}, \boldsymbol{x}, \gamma k) d S_{\xi},
\end{array}\right\} \boldsymbol{x} \in S_{\epsilon},
$$

written respectively for the exterior and interior region in terms of the scattered field over $S_{\epsilon}$. Here $\kappa(\boldsymbol{x})$ is the free term, equal to $\frac{1}{2}$ if $B_{\epsilon}$ is smooth at $\boldsymbol{x}$, and $\boldsymbol{n}$ is again oriented outward from $\Omega^{-}$. One may note that integral equations (17) are free of Cauchy principal values owing to the particular structure of the acoustic fundamental solution and the earlier assumption that $S_{\epsilon}$ is piecewise-smooth with components of class $C^{2}$ [6].

In this setting, the limiting form of $p^{\epsilon}$ and $\nabla p^{\epsilon}$ in $B_{\epsilon}$ as $\epsilon \rightarrow 0$ is sought by performing an asymptotic analysis of (17), following e.g. the procedures described in [3], and the invoking interfacial conditions $(3 \mathrm{~d})$, to obtain

$$
p^{\epsilon}(\boldsymbol{\xi})=p^{\mathrm{F}}\left(\boldsymbol{x}^{\circ}\right)+o(1), \quad \nabla p^{\epsilon}(\boldsymbol{\xi})=\nabla p^{\mathrm{F}}\left(\boldsymbol{x}^{\circ}\right) \cdot \nabla_{\zeta} \boldsymbol{g}(\boldsymbol{\zeta})+o(1), \quad \boldsymbol{\xi} \in B_{\epsilon}, \quad \boldsymbol{\zeta} \in \mathscr{B} .
$$

Here $\boldsymbol{\zeta}=\left(\boldsymbol{\xi}-\boldsymbol{x}^{\circ}\right) / \epsilon$ is the scaled position vector, and $\boldsymbol{g}(\boldsymbol{\zeta})$ is a component of the vector solution pair $(\boldsymbol{g}, \tilde{\boldsymbol{g}})$ that satisfies the (vector) Laplace transmission problem for the "unit" obstacle $\mathscr{B}=\left.\mathscr{B}_{\epsilon}\right|_{\epsilon=1}$ embedded in an infinite medium:

$$
\begin{gathered}
\nabla_{\zeta}^{2} \boldsymbol{g}=\mathbf{0} \quad(\boldsymbol{\zeta} \in \mathscr{B}), \quad \nabla_{\zeta}^{2} \tilde{\boldsymbol{g}}=\mathbf{0} \quad\left(\boldsymbol{\zeta} \in \mathbb{R}^{3} \backslash \mathscr{B}\right), \\
\tilde{\boldsymbol{g}}+\boldsymbol{\zeta}=\boldsymbol{g}, \quad \tilde{\boldsymbol{g}}_{, \eta}+\boldsymbol{\eta}=\beta \boldsymbol{g}_{, \eta}, \quad(\boldsymbol{\zeta} \in \mathscr{S}) .
\end{gathered}
$$

In (19), $\boldsymbol{\eta}(\boldsymbol{\zeta})=\boldsymbol{n}(\boldsymbol{\xi})$ is the unit normal on $\mathscr{S}$, oriented toward the interior of $\mathscr{B}$; the normal derivatives $\tilde{\boldsymbol{g}}_{, \eta}$ and $\boldsymbol{g}_{, \eta}$ are both defined in terms of $\boldsymbol{\eta}$, and the exterior field $\tilde{\boldsymbol{g}}$ decays as $O\left(|\zeta|^{-1}\right)$ or faster at infinity.

On substituting (18) into (16), one finds that the scattered field for a vanishing inclusion can be expressed as

$$
\begin{aligned}
& \tilde{p}^{\epsilon}(\boldsymbol{x})=\epsilon^{3}|\mathscr{B}|\left\{(1-\beta) \nabla p^{\mathrm{F}}\left(\boldsymbol{x}^{\circ}\right) \cdot \boldsymbol{A} \cdot \nabla \hat{G}\left(\boldsymbol{x}^{\circ}, \boldsymbol{x}, k\right)\right. \\
&\left.\quad-\left(1-\beta \gamma^{2}\right) k^{2} p^{\mathrm{F}}\left(\boldsymbol{x}^{\circ}\right) \hat{G}\left(\boldsymbol{x}^{\circ}, \boldsymbol{x}, k\right)\right\}+o\left(\epsilon^{3}\right), \quad \boldsymbol{x} \in \Omega_{\epsilon}^{-}, \quad \epsilon \rightarrow 0
\end{aligned}
$$

where $\boldsymbol{A}$ is the (constant, second-order) polarization tensor

$$
\boldsymbol{A}=|\mathscr{B}|^{-1} \int_{\mathscr{B}} \nabla_{\zeta} \boldsymbol{g} d V_{\zeta}=-|\mathscr{B}|^{-1} \int_{\mathscr{S}} \boldsymbol{g} \otimes \boldsymbol{\eta} d S_{\zeta},
$$

whose latter expression stems from the divergence theorem. On the basis of (19) and (21), it is noted that the polarization tensor depends only on the shape $\mathscr{B}$ and relative density $\beta$ of the nucleating obstacle. 
One may also observe from (20) that $\tilde{p}^{\epsilon}(\boldsymbol{x})$ behaves as $O\left(\epsilon^{3}\right)$ in $\Omega^{-}$as $\epsilon \rightarrow 0$ and thus, by way of (14), $h(\epsilon) \propto \epsilon^{3}$ regardless of the shape of $B_{\epsilon}$. Since the choice of the multiplicative constant is, through (12), arbitrary, it is assumed in this study that

$$
h(\epsilon)=\epsilon^{3}|\mathscr{B}|,
$$

i.e. that $h(\epsilon)$ is given by the measure of a vanishing obstacle domain. On the basis of (20) and (22), the formula for topological derivative (14) reduces to

$$
\begin{aligned}
& \mathcal{T}\left(\boldsymbol{x}^{\circ}, \beta, \gamma ; f\right)=\int_{\Gamma^{\mathrm{obs}}} \operatorname{Re}\left\{\frac { \partial \varphi } { \partial p } ( p ^ { \mathrm { F } } ( \boldsymbol { \xi } ) , \boldsymbol { \xi } ) \left[(1-\beta) \nabla p^{\mathrm{F}}\left(\boldsymbol{x}^{\circ}\right) \cdot \boldsymbol{A} \cdot \nabla \hat{G}\left(\boldsymbol{x}^{\circ}, \boldsymbol{\xi}, k\right)\right.\right. \\
&\left.\left.-\left(1-\beta \gamma^{2}\right) k^{2} p^{\mathrm{F}}\left(\boldsymbol{x}^{\circ}\right) \hat{G}\left(\boldsymbol{x}^{\circ}, \boldsymbol{\xi}, k\right)\right]\right\} d S_{\xi}, \quad \boldsymbol{x}^{\circ} \in \Omega,
\end{aligned}
$$

where, for the least-squares-type cost functional (6),

$$
\frac{\partial \varphi}{\partial p}\left(p^{\mathrm{F}}(\boldsymbol{\xi}), \boldsymbol{\xi}\right)=W(\boldsymbol{\xi})\left(\overline{p^{\mathrm{F}}(\boldsymbol{\xi})-p^{\mathrm{obs}}(\boldsymbol{\xi})}\right) .
$$

Here it is noted that (23) consists of a dipole and a monopole term, which vanish respectively when the mass density and compressibility of the obstacle equals that of the background medium. The limiting case of an acoustically-hard vanishing obstacle (exterior Neumann problem) is obtained by taking the limit $\rho^{*} \rightarrow \infty$, i.e. by setting $\beta=0$ in (23) (see also [14] in the context of general acoustics). In contrast, the case of an acoustically-soft vanishing obstacle $(\beta \rightarrow \infty)$ leads to an asymptotic behavior that is distinct from (23) and hence not recoverable by the present approach. The key reason for such distinction is that the asymptotic analysis underpinning (20) and (22) is not appropriate when the Dirichlet condition is assumed along the boundary of a vanishing obstacle.

A generalization of (5) and (23) to situations involving $Q$ sequentially-applied acoustic sources $f^{q}$ is straightforward and involves external summation of the form

$$
\mathcal{J}_{f}(\cdot) \equiv \sum_{q=1}^{Q} \mathcal{J}\left(\cdot ; f^{q}\right) \quad \text { and } \quad \mathcal{T}_{f}(\cdot) \equiv \sum_{q=1}^{Q} \mathcal{T}\left(\cdot ; f^{q}\right)
$$

\subsection{Explicit Expressions}

In what follows, explicit expressions for generalized topological sensitivity are given for the canonical cases of spherical and ellipsoidal inclusions. By their nature, the ensuing results are closely related to the low-frequency approximation of the acoustic scattering problem, a subject dating back to [29] and more recently covered in [14].

Spherical obstacle The simplest specialization of the foregoing developments is the case when the vanishing obstacle is spherical, i.e. when $\mathscr{S}$ is the unit sphere and $|\mathscr{B}|=4 \pi / 3$. For this geometry, (19) can be easily solved using spherical harmonics, to obtain

$$
\boldsymbol{g}(\boldsymbol{\zeta})=\frac{3}{\beta+2} \boldsymbol{\zeta}, \quad \boldsymbol{\zeta} \in \mathscr{B} .
$$


The polarization tensor (21) is accordingly found, on the basis of (25), as

$$
\boldsymbol{A}^{\mathrm{sph}}=\frac{3}{\beta+2} \boldsymbol{I}_{2}
$$

where $\boldsymbol{I}_{2}$ is the second-order identity tensor. It is interesting to note that in dealing with 3D electromagnetic scattering, [35] and [1] list apparently inconsistent expressions for the polarization tensor, expressible respectively as

$$
\boldsymbol{A}^{\mathrm{sph}}=2 \frac{\beta-1}{2 \beta+1} \boldsymbol{I}_{2}, \quad \boldsymbol{A}^{\mathrm{sph}}=8 \pi \frac{1}{\beta+1} \boldsymbol{I}_{2},
$$

using present notation. On comparing (19) in this paper with the last formula on page 195 in [35] and the last formula on page 883 in [1], it can be shown that $\beta$ in (27) takes the meaning of either relative magnetic permeability $\mu^{\star} / \mu$ or relative electric permittivity $\epsilon^{\star} / \epsilon$.

Ellipsoidal obstacle To examine the behavior of (23) when the vanishing obstacle is non-spherical, one may consider the solution to the Laplace transmission problem (19) in situations when $\mathscr{S}$ is an ellipsoid with principal directions $\boldsymbol{e}_{1}^{\prime}, \boldsymbol{e}_{2}^{\prime}, \boldsymbol{e}_{3}^{\prime}$ and semi-axes $a_{1}, a_{2}, a_{3}$. Here the characteristic volume is $|\mathscr{B}|=4 \pi a_{1} a_{2} a_{3} / 3$ which is inherently accounted for through the definition of $h(\epsilon)$. In this setting, the analytical solution of (19) is sought by introducing an ellipsoidal coordinate system and expanding $\boldsymbol{g}, \tilde{\boldsymbol{g}}$ in terms of spheroidal harmonics [23]. This procedure yields the solution for $\boldsymbol{g}$ as

$$
\boldsymbol{g}(\boldsymbol{\zeta})=\sum_{m=1}^{3} \frac{1}{1-(1-\beta) I_{m}}\left(\boldsymbol{e}_{m}^{\prime} \otimes \boldsymbol{e}_{m}^{\prime}\right) \cdot \boldsymbol{\zeta}, \quad \boldsymbol{\zeta} \in \mathscr{B}
$$

where $I_{m}$ are the functions of the two aspect ratios of $\mathscr{B}\left(\right.$ e.g. $a_{2} / a_{1}$ and $\left.a_{3} / a_{1}\right)$ given by

$$
I_{m}=\frac{a_{1} a_{2} a_{3}}{2} \int_{0}^{\infty} \frac{d r}{\left(r+a_{m}^{2}\right) \sqrt{r+a_{1}^{2}} \sqrt{r+a_{2}^{2}} \sqrt{r+a_{3}^{2}}}, \quad m=1,2,3
$$

On the basis of (18) and (28), the polarization tensor featured in (20) takes the form

$$
\boldsymbol{A}^{\mathrm{ell}}=\sum_{m=1}^{3} \frac{\boldsymbol{e}_{m}^{\prime} \otimes \boldsymbol{e}_{m}^{\prime}}{1-(1-\beta) I_{m}}
$$

that is diagonal in the principal axes frame $\left(\boldsymbol{e}_{1}^{\prime}, \boldsymbol{e}_{2}^{\prime}, \boldsymbol{e}_{3}^{\prime}\right)$, albeit not isotropic as in (26).

To demonstrate that $\boldsymbol{A}^{\text {ell }}$ remains finite for arbitrarily chosen relative inclusion density $\beta \geq 0$ and semi-axes $a_{1}, a_{2}, a_{3}$, it can be shown by elementary calculations that $I^{(1)}+I^{(2)}+I^{(3)}=1$ which, through restriction $I_{m}>0$, yields $I_{m}<1$ and thus the desired result. Moreover, it is worth noting that on setting $a_{1}=a_{2}=a_{3}=1$ in (28), one obtains $I_{m}=1 / 3(m=1,2,3)$ which directly reduces the polarization tensor (29) to its spherical counterpart (26). Depending on the quantity of interest, however, such a reduction in the context of acoustic scattering may not always involve a trivial calculation [14]. 


\subsection{Qualitative obstacle identification via generalized topological sensitivity}

Equation (23) provides, when applied to the featured cost functional $\mathcal{J}$, an information indicating whether $\mathcal{J}$ increases $\left(\mathcal{T}\left(\boldsymbol{x}^{\circ}\right)>0\right)$ or decreases $\left(\mathcal{T}\left(\boldsymbol{x}^{\circ}\right)<0\right)$ in response to the nucleation at $\boldsymbol{x}^{\circ}$ of an infinitely small penetrable scatterer with prescribed shape and material characteristics. Thus, the topological sensitivity field $\mathcal{T}\left(\boldsymbol{x}^{\circ}\right)$ is guaranteed to define a correct obstacle indicator only in the limit as the size of a hidden obstacle approaches zero. It is nonetheless natural to examine whether the spatial distribution of $\mathcal{T}$ can still furnish a useful information for the reconstruction and characterization of finite obstacles. Motivated by the ultimate goal of minimizing $\mathcal{J}$, the key idea in this setting is to approximate the support of a hidden (finite) obstacle via regions where $\mathcal{T}$ attains pronounced negative values. While the reasoning behind such an idea is heuristic as it lacks a rigorous mathematical backing such as that underpinning the linear sampling method $[11,10]$, the numerical experiments presented in this study as well as the other (impenetrable obstacle) results in earlier works $[20,8,18,17]$ support the utility of the proposed notion as a preliminary reconstruction tool. Since the computation of the indicator function $\mathcal{T}$ (over the volume of interest) is significantly faster than an iterative (e.g. minimization-based) inversion, this approach offers a convenient means of extracting preliminary obstacle information from the data $p^{\text {obs }}$. Such information can then be used either in a stand-alone manner for limited-accuracy reconstruction, or as a reliable initial "guess" for more elaborate, iterative reconstruction schemes.

\section{Discussion}

For a broader perspective of the foregoing results, this section deals with complementary developments that include a discussion of the polarization tensor, an adjoint-field approach to topological sensitivity, and a treatment of finite background domains.

\subsection{Nature of the polarization tensor}

With the help of the Green's first identity applied to harmonic fields $\tilde{g}_{k}$ in $\mathbb{R}^{3} \backslash \overline{\mathscr{B}}, g_{k}$ in $\mathscr{B}$, and $\zeta_{k}$ in $\mathscr{B}(k=1,2,3)$, where $\boldsymbol{g}=g_{k} \boldsymbol{e}_{k}$ and $\tilde{\boldsymbol{g}}=\tilde{g}_{k} \boldsymbol{e}_{k}$ are the respective interior and exterior parts of the solution to the Laplace problem (19), one obtains the identity

$$
\int_{\mathbb{R}^{3} \backslash \mathscr{B}} \tilde{g}_{i, k} \tilde{g}_{j, k} d V_{\zeta}+\int_{\mathscr{B}}\left[\beta g_{i, k} g_{j, k}+\delta_{i k}\right] d V_{\zeta}=\int_{\mathscr{S}}\left[\tilde{g}_{i} \tilde{g}_{j, \eta}-\beta g_{i} g_{j, \eta}-\zeta_{i} \eta_{j}\right] d S_{\zeta} .
$$

On the basis of the interfacial conditions in (19), (21), (30), and the Green's second identity

$$
\int_{\mathscr{S}} \zeta_{i} g_{j, \eta} d S_{\zeta}=\int_{\mathscr{S}} \eta_{i} g_{j} d S_{\zeta}
$$

one finds that the Cartesian components of $\boldsymbol{A}$ are such that

$$
A_{i j}+\beta A_{j i}=\int_{\mathbb{R}^{3} \backslash \mathscr{B}} \tilde{g}_{i, k} \tilde{g}_{j, k} d V_{\zeta}+\int_{\mathscr{B}}\left[\beta g_{i, k} g_{j, k}+\delta_{i k}\right] d V_{\zeta}
$$


The right-hand side of (31) clearly defines a symmetric and positive-definite secondorder tensor. Hence one must have $A_{i j}+\beta A_{j i}=A_{j i}+\beta A_{i j}$, i.e. $A_{i j}=A_{j i}(\beta \neq 1)$. Equation (31) thus shows that $(1+\beta) \boldsymbol{A}$, and hence the polarization tensor $\boldsymbol{A}$, is symmetric and positive-definite regardless of the shape of $\mathscr{B}$. This is a useful result as it indicates that the leading-term scattering effects (20), and thus the topological sensitivity (23), for a connected infinitesimal obstacle of arbitrary shape might be cast in terms of its ellipsoidal counterpart. Whether such a possibility can be materialized, however, depends on the solvability of the non-linear system

$$
(1-\beta) I_{m}\left(\frac{a_{2}}{a_{1}}, \frac{a_{3}}{a_{1}}\right)=1-\lambda_{m}^{-1}, \quad m=1,2,3
$$

in terms of parameters $a_{2} / a_{1}, a_{3} / a_{1}$ and $\beta$ of an equivalent-ellipsoidal vanishing obstacle where $\lambda_{m}$ are the (real-valued) eigenvalues of $\boldsymbol{A}$.

\subsection{Direct versus adjoint field formulation}

For generality it is instructive to recast (23), obtained via the so-called direct approach, within the framework of the adjoint field method that is commonly used in the shape sensitivity (e.g. [5, 22]) and topological sensitivity (e.g. [19]) analyses. In the present setting, the adjoint field is defined as an auxiliary pressure field

$$
\stackrel{\star}{p}(\boldsymbol{x})=\int_{\Gamma^{\mathrm{obs}}} \frac{\partial \varphi}{\partial p}\left(p^{\mathrm{F}}(\boldsymbol{\xi}), \boldsymbol{\xi}\right) \hat{G}(\boldsymbol{x}, \boldsymbol{\xi}, k) d S_{\xi},
$$

signifying the response of the obstacle-free domain $\Omega$ due to virtual excitation

$$
g(\boldsymbol{\xi})=\frac{\partial \varphi}{\partial p}\left(p^{\mathrm{F}}(\boldsymbol{\xi}), \boldsymbol{\xi}\right), \quad \boldsymbol{\xi} \in \Gamma^{\mathrm{obs}},
$$

written in terms of the misfit function $\varphi$ for $p=p^{\mathrm{F}}$. On the basis of (14), (20), (33) and (34), an adjoint-field variant of formula (23) for material-topological sensitivity is obtained as

$$
\mathcal{T}\left(\boldsymbol{x}^{\circ}, \beta, \gamma ; f\right)=\operatorname{Re}\left\{(1-\beta) \nabla p^{\mathrm{F}} \cdot \boldsymbol{A} \cdot \nabla \stackrel{\star}{p}-\left(1-\beta \gamma^{2}\right) k^{2} p^{\mathrm{F}} \stackrel{\star}{p}\right\}\left(\boldsymbol{x}^{\circ}\right), \quad \boldsymbol{x}^{\circ} \in \Omega .
$$

One may note that the difference between (23) and (35) is at this point strictly formal. In particular, for all background domains $\Omega$ for which the Green's function is available, expressions (23) and (35) lead to the same Green's function-based computational scheme for the evaluation of $\mathcal{T}$. For more complex forms of $\Omega$ (e.g. finite domains of arbitrary shape), on the other hand, practical unavailability of suitable Green's function requires a treatment wherein the direct and adjoint field formulations lead to distinct computational procedures. This issue is addressed next.

\subsection{Finite background domains}

To deal with acoustic obstacle reconstruction in the context of finite bodies, consider a background domain $\Omega$ with properties $(c, \rho)$ and external boundary $\Sigma=\partial \Omega$, housing 
a penetrable obstacle $B$ with properties $\left(c^{*}, \rho^{*}\right)$. Following the previously established convention, $\Omega^{-}=\Omega \backslash B, S=\partial B, \bar{\Omega}^{-}=\Omega^{-} \cup \Sigma \cup S$, while $\boldsymbol{n}$ and $\boldsymbol{n}^{\prime}$ denote the respective normals on $\Sigma \cup S$ (oriented outward from $\Omega^{-}$) and $\Gamma_{\Omega}^{\text {obs }}$. On writing $\Sigma=\Sigma_{\mathrm{D}} \cup \Sigma_{\mathrm{N}}$, it is assumed that $\Omega^{-}$is subject to a domain source distribution and to Dirichlet and Neumann boundary data prescribed respectively over $\Sigma_{\mathrm{D}}$ and $\Sigma_{\mathrm{N}}$. Next, let the Green's function $\check{G}$ solve (8) over $\Omega$ subject to homogeneous boundary conditions

$$
\check{G}(\boldsymbol{\xi}, \boldsymbol{x}, k)=0 \quad\left(\boldsymbol{\xi} \in \Sigma_{\mathrm{D}}\right), \quad \check{H}(\boldsymbol{\xi}, \boldsymbol{x}, k)=0 \quad\left(\boldsymbol{\xi} \in \Sigma_{\mathrm{N}}\right) .
$$

On superseding $\hat{G}$ and $\hat{H}$ respectively by $\check{G}$ and $\check{H}$, the scattered field $\tilde{p}^{\epsilon}$ is, for a finite body $\Omega_{\epsilon}^{-}$, still given by the integral representation (16) and the asymptotic expansion (20) still holds [3] with the polarization tensor $\boldsymbol{A}$ again given by (21) and (19). As a result, expression (23) for generalized topological sensitivity (where $\hat{G}$ is replaced with $\check{G}$ ) and its adjoint-field variant (35) still hold.

The analytical expression of the latter Green's function is, however, not available except for very few geometrically-simple domains $\Omega$. Assuming that the polarization tensor $\boldsymbol{A}$ and the free field $p^{\mathrm{F}}$ are calculated beforehand, the computation of topological derivative for a finite body using the direct approach (23) consequently entails solving one boundary value problem per sampling point $\boldsymbol{x}^{\circ}$ (as a means to evaluate the weighted sum of $\check{G}\left(\boldsymbol{x}^{\circ}, \boldsymbol{\xi}, k\right)$ and $\left.\nabla \check{G}\left(\boldsymbol{x}^{\circ}, \boldsymbol{\xi}, k\right)\right)$.

This potentially demanding computational procedure can be significantly reduced by resorting to the adjoint field approach. Indeed, upon invoking the definition of $\check{G}(\boldsymbol{\xi}, \boldsymbol{x}, k)$ and the symmetry property $\check{G}(\boldsymbol{x}, \boldsymbol{\xi}, k)=\check{G}(\boldsymbol{\xi}, \boldsymbol{x}, k)$, one can show that the adjoint field $\stackrel{\star}{p}(\boldsymbol{x})$, given by $(33)$, solves the transmission problem

$$
\begin{aligned}
& \nabla^{2} \stackrel{p}{p}+k^{2} \stackrel{p}{p}=0 \quad\left(\boldsymbol{\xi} \in \Omega \backslash \Gamma_{\Omega}^{\mathrm{obs}}\right), \\
& {\left[\left[{ }^{\star}\right]\right]=0, \quad\left[\left[{\stackrel{p}{p}, n^{\prime}}^{\prime}\right]\right]=g \quad\left(\boldsymbol{\xi} \in \Gamma_{\Omega}^{\mathrm{obs}}\right),} \\
& \stackrel{\star}{p}=0 \quad\left(\boldsymbol{\xi} \in \Sigma_{\mathrm{D}}\right), \quad \stackrel{\star}{p}_{, n}=0 \quad\left(\boldsymbol{\xi} \in \Sigma_{\mathrm{N}} \backslash \Gamma_{\Sigma}^{\mathrm{obs}}\right), \\
& \stackrel{\star}{p}_{, n^{\prime}}=g \quad\left(\boldsymbol{\xi} \in \Gamma_{\Sigma}^{\mathrm{obs}}\right),
\end{aligned}
$$

where the single-layer excitation $g$ is given by $(34), \boldsymbol{n}^{\prime}$ is the unit normal on $\Gamma_{\Omega}^{\text {obs }}$, and $[[w]]=\lim _{\tau \rightarrow 0} w\left(\boldsymbol{\xi}+\tau \boldsymbol{n}^{\prime}\right)-w\left(\boldsymbol{\xi}-\tau \boldsymbol{n}^{\prime}\right), \boldsymbol{\xi} \in \Gamma_{\Omega}^{\text {obs }}$ denotes the jump in $w$ across $\Gamma_{\Omega}^{\text {obs }}$. Hence, the adjoint field approach (35) requires the solution of only one additional boundary value problem (used to compute $\stackrel{\star}{p}$ and $\nabla \stackrel{\star}{p}$ ) that is common for all sampling points. In this sense, the adjoint field methodology represents the most effective (if not direct) route for computing the topological sensitivity in a general background-body configuration. As such, it will be used as a computational basis for the ensuing examples.

\section{Results}

To examine the effectiveness of generalized topological sensitivity as a tool for preliminary obstacle reconstruction and identification, a set of numerical results is presented next. In the sequel, each synthetic testing configuration consists of $M$ measurement points $\boldsymbol{x}^{m}(m=1, \ldots, M)$. For reconstruction purposes, the obstacle 
(or a set thereof) is illuminated by a set of $Q$ point sources of magnitude $\rho c^{2} \ell$, applied in sequence at locations $\boldsymbol{x}^{q}(q=1, \ldots, Q)$ where $\ell$ is the reference length. On the basis of (5), (6) and (24), the generalized topological sensitivity is thus computed with reference to the least-squares cost function

$$
\mathcal{J}_{f}\left(\Omega^{-}, \beta, \gamma\right)=\frac{1}{2} \sum_{q=1}^{Q} \sum_{m=1}^{M}\left\{p_{q}\left(\boldsymbol{x}^{m}\right)-p_{q}^{\mathrm{obs}}\left(\boldsymbol{x}^{m}\right)\right\} \overline{\left\{p_{q}\left(\boldsymbol{x}^{m}\right)-p_{q}^{\mathrm{obs}}\left(\boldsymbol{x}^{m}\right)\right\}}
$$

where $p_{q}^{\text {obs }}$ and $p_{q}$ denote respectively the measured (i.e. "true") and trial acoustic fields induced at sensor locations $\boldsymbol{x}^{m}$ by the source acting at $\boldsymbol{x}^{q}$.

In what follows, the synthetic data $p_{q}^{\text {obs }}$ in (37) are generated for five "true" obstacle configurations: i) one penetrable obstacle $\left(\beta^{\text {true }}=2, \gamma^{\text {true }}=0.5\right)$; ii) one rigid obstacle $\left(\beta^{\text {true }}=0, \gamma^{\text {true }}=1\right)$; iii $)$ two penetrable obstacles; iv) two rigid obstacles, and $\left.\mathrm{v}\right)$ two dissimilar obstacles, one penetrable and one rigid. For brevity, these configurations are labeled as P, R, PP, RR and PR, respectively. All obstacles are ellipsoidal, their semiaxes being aligned with the reference Cartesian frame as shown in Fig. 2. The geometric and material parameters for all five obstacle configurations are listed in Table 1. With the exception of three-dimensional plots, all ensuing $\mathcal{T}_{f}$-distributions are plotted with reference to the "vertical" plane $\xi_{2}=0.5 \ell$. Accordingly, the horizontal and vertical axes of sectional distributions in Figs. $3-8$ and $10-14$ carry implicit labels $\xi_{1} / \ell$ and $\xi_{3} / \ell$, respectively. To provide a basis for comparison, intersection of the featured plane $\left(\xi_{2}=0.5 \ell\right)$ with "true" scatterers is indicated in white in the figures.

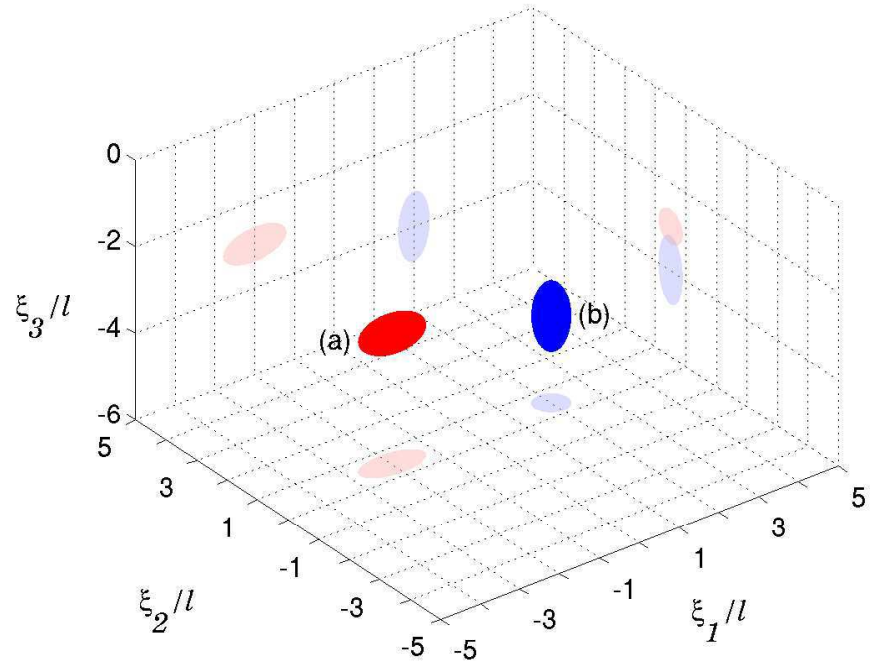

Figure 2. True obstacle configurations $\mathrm{P}(\mathrm{a}), \mathrm{R}(\mathrm{b})$, and PR (a-b).

The scatterers are embedded in either acoustic half-space $(\mathrm{H})$ or the full-space (F). Building upon the previously adopted notation, the scatterer-background medium combinations are designated by a hyphenated symbol, with e.g. P-F denoting a single penetrable obstacle in the acoustic full-space. The synthetic data $p_{q}^{\text {obs }}$ are computed by means of a direct boundary element method (BEM), wherein each ellipsoidal obstacle 
is meshed using 384 eight-node elements. In this way, the BEM meshes feature at least 15 nodes per wavelength for the highest wavenumber considered, $k \ell=8$.

The "complete" (i.e. the most extensive) testing configuration used in the examples consists of an array of points located at the vertices of six uniform $10 \times 10$ rectangular grids placed along the faces of a box defined by the planes $\xi_{1}= \pm 6 \ell, \xi_{2}= \pm 6 \ell$ and $\xi_{3}=0,-6 \ell$. This testing array, henceforth referred to as $T_{6}$, is used for both source and sensor locations, so that $Q=M=602$ in this case. For clarity, it is worth noting that the three-dimensional region in Fig. 2 is that enclosed by $T_{6}$.

In what follows, the topological sensitivity is evaluated at the nodes of a uniform sampling grid $\mathcal{G}$ consisting of $51 \times 51=2,601$ points in the $2 \mathrm{D}$ (sectional) diagrams and $51 \times 51 \times 51=132,651$ points in the $3 \mathrm{D}$ plots. The grid samples the region $-5 \ell \leq \xi_{1}, \xi_{2} \leq 5 \ell,-5.5 \ell \leq \xi_{3} \leq-0.5 \ell$, i.e. most of the cubical enclosed by testing configuration $T_{6}$. For the highest wavenumber considered $(k \ell=8)$, the horizontal and vertical spacing of the sampling grid are approximately $1 / 4$ and $1 / 8$ of the wavelength, respectively. Except for the results shown in Fig. 7, the topological sensitivity field is computed on the basis of a spherical nucleating inclusion shape $\mathscr{B}$. All computations, including the BEM used for the creation of synthetic data, are implemented within the MATLAB environment and run on a laptop computer with a $1.7 \mathrm{MHz}$ Pentium IV processor. On this platform, computation of $\mathcal{T}_{f}$ over the entire $3 \mathrm{D}$ sampling grid $\mathcal{G}\left(132,651\right.$ points) for testing configuration $T_{6}$ in the acoustic half-space case takes about 30 minutes of CPU time, with the BEM calculation of synthetic data requiring approximately 15 additional minutes for the dual-scatterer configurations.

\subsection{Influence of testing grid aperture}

Testing configuration $T_{6}$, defined earlier, is deemed to be of full aperture inasmuch as the source and sensor locations completely surround the region of interest. To study the effects of limited aperture, two other testing configurations, $T_{1}$ and $T_{2}$, are considered. Configuration $T_{1}$ consists of a uniform $(10 \times 10)$ square "source" grid of size $10 \ell$ with $11 \times 11$ source points, and a uniform $(20 \times 20)$ square "receiver" grid of size $10.5 \ell$ with $22 \times 22$ sensor locations, both grids lying in the plane $\xi_{3}=0$ and centered at $\xi_{1}=\xi_{2}=0$. Configuration $T_{2}=T_{1} \cup T_{1}^{\prime}$, where $T_{1}^{\prime}$ is obtained by translating $T_{1}$ in the amount of $6 \ell$ in the negative $\xi_{3}$-direction. Accordingly, $(Q, M)=(121,484)$ for $T_{1}$ and $(242,968)$ for $T_{2}$.

Figures 3 and 4 shows the distribution of $\mathcal{T}_{f}\left(\boldsymbol{x}^{\circ}, \beta^{\text {true }}, \gamma^{\text {true }}\right)$ in the vertical plane

Table 1. Scatterer configurations.

\begin{tabular}{|c|c|c|c|c|c|}
\hline Name & $\mathrm{P}$ & $\mathrm{R}$ & $\mathrm{PP}$ & $\mathrm{RR}$ & $\mathrm{PR}$ \\
\hline $\begin{array}{c}\text { Properties } \\
\left(\beta^{\text {true }}, \gamma^{\text {true }}\right)\end{array}$ & $(2,0.5)$ & $(0,1)$ & $(2,0.5)$ & $(0,1)$ & $(2,0.5)$ \\
\hline $\begin{array}{c}\text { Centroid } \\
\boldsymbol{x}_{c}^{\text {true }} / \ell\end{array}$ & $(-2,0.5,-3)$ & $(2,0.5,-4)$ & $(2,0.5)$ & $(0,1)$ & $(0,1)$ \\
\hline $\begin{array}{c}\text { Semi-axes } \\
\mathbf{a}^{\text {true }} / \ell\end{array}$ & $(0.8,0.4,0.4)$ & $(0.4,0.4,0.8)$ & $(2,0.5,-4)$ & $\left(\begin{array}{c}0.8,0.4,0.4) \\
(2,0.5,-4)\end{array}\right.$ & $\begin{array}{c}(0.8,0.4,0.5,0.4) \\
(2,0.5,-4)\end{array}$ \\
\hline
\end{tabular}


$\xi_{2}=0.5 \ell$, computed over the $51 \times 51$ uniform sampling grid for the testing arrangements $T_{1}, T_{2}$ and $T_{6}$, wavenumbers $k \ell=2,4,8$, and scatterer configurations $\mathrm{P}-\mathrm{F}$ (Fig. 3) and $\mathrm{P}-\mathrm{H}$ (Fig. 4). It is not surprising that the best delineation of the obstacle for both configurations is obtained for $T_{6}$, i.e. the case of full aperture. The differences between the images obtained under the respective conditions of full-space and halfspace background domains are highlighted by the detail in Fig. 5 . In the display, the distribution of $\mathcal{T}_{f}\left(\boldsymbol{x}^{\circ}, \beta^{\text {true }}, \gamma^{\text {true }}\right)$ is seen to provide a good delineation of the scatterer shape for the full-space case, especially for $k \ell=8$. In contrast, the distribution of $\mathcal{T}_{f}\left(\boldsymbol{x}^{\circ}, \beta^{\text {true }}, \gamma^{\text {true }}\right)$ for the half-space case is skewed towards the "top" surface $\xi_{3}=0$.

On denoting the largest dimension of the scatterers by $L=1.6 \ell$, one may note that the approximate wavelengths of "illuminating" waves featured in Figs. 3 and 4 are $4 L, 2 L, L$ and $0.5 L$ for $k \ell=1,2,4$ and 8 , respectively. In particular, the wavelengths for $k \ell=1,2$ and 4 are larger than or of a comparable size to the diameter of the scatterer and can thus be categorized as belonging to the so-called resonance region [12].

\subsection{Influence of testing grid density}

With reference to the half-space scatterer configuration $\mathrm{P}-\mathrm{H}$ and the full-aperture testing arrangement $T_{6}$, the distributions of $\mathcal{I}_{f}\left(\boldsymbol{x}^{\circ}, \beta^{\text {true }}, \gamma^{\text {true }}\right)$ are computed for three densities of source and measurement grids, namely $T_{6}(2), T_{6}(5)$ and $T_{6}(10) \equiv T_{6}$ and plotted in Fig. 6. Here $T_{6}(2)$ and $T_{6}(5)$ are defined in the same way as $T_{6}$ where $Q=M=602$ but on the basis of $2 \times 2(Q=M=26)$ and $5 \times 5(Q=M=152)$ rectangular grids on each face, respectively. From the display, one may note that the coarser grids $T_{6}(2)$ and $T_{6}(5)$ still provide acceptable results (with the notable exception of $T_{6}(2)$ for $k \ell=8$ ) and in particular better than those obtained using "dense" but partial-aperture grids $T_{1}$ and $T_{2}$, despite the larger number of source and sensor points in the latter two cases. These results suggest that the testing aperture is a more critical factor than the grid density for quality of obstacle delineation on the basis of topological sensitivity distribution.

\subsection{Nucleating obstacle: influence of shape}

In assessing the effectiveness of generalized topological sensitivity as a tool for preliminary obstacle reconstruction, a practical question arises as to the effect of the (assumed) shape $\mathscr{B}$ of a nucleating obstacle, manifested via tensor $\boldsymbol{A}$ in (35), on the spatial distribution of $\mathcal{T}_{f}$. This influence is illustrated in Fig. 7 which shows the distribution of $\mathcal{T}_{f}\left(\boldsymbol{x}^{\circ}, \beta^{\text {true }}, \gamma^{\text {true }}\right)$ in the vertical plane $\xi_{2}=0.5 \ell$, on the basis of the testing arrangement $T_{6}$, for three reference shapes $\mathscr{B}$ : sphere $\left(a_{1}=a_{2}=a_{3}\right)$, "vertical" ellipsoid $\left(2 a_{1}=2 a_{2}=a_{3}\right)$, and "horizontal" ellipsoid $\left(a_{1}=2 a_{2}=2 a_{3}\right)$, where $a_{1}, a_{2}$ and $a_{3}$ denote again the ellipsoid semi-axes. As can be seen from the display, the shape of $\mathscr{B}$ has a significant effect on the overall magnitude of $\mathcal{T}_{f}$, but not on its relative distribution. This observation is supported by the similar results obtained for other obstacle combinations, omitted here for brevity. Since it is the relative distribution of $\mathcal{T}_{f}$ that provides most information about the support of the obstacle, one of the conclusions of this study is 
that the choice of $\mathscr{B}$ is not a critical component of the proposed (qualitative) imaging technique. This conclusion, however, may not hold for procedures where higher-order terms are considered in the expansion (11), which may furnish more accurate information about the geometry of the scatterer (see [7] for impenetrable obstacles).

\subsection{Nucleating obstacle: influence of constitutive parameters}

In Figs. 3-7, the $\mathcal{T}_{f}$-distribution is calculated assuming "correct" material parameters for the nucleating obstacle, namely $(\beta, \gamma)=\left(\beta^{\text {true }}, \gamma^{\text {true }}\right)$. The aim of this section is to examine the effect of material constants $\beta$ and $\gamma$ on the generalized topological sensitivity, and whether considering the $\mathcal{T}_{f}$-distributions with spatially-varying $(\beta, \gamma)$ allows one to obtain information about the constitutive nature of the true scatterer.

With reference to the half-space scatterer configuration $\mathrm{P}-\mathrm{H}$, the distribution of $\mathcal{T}_{f}\left(\boldsymbol{x}^{\circ}, \beta^{\text {true }}, \gamma^{\text {true }}\right)$ is first compared in Fig. 8 to $\mathcal{T}_{f}\left(\boldsymbol{x}^{\circ}, 0, \gamma^{\text {true }}\right)$ obtained on the basis of a rigid infinitesimal obstacle. As mentioned earlier, all simulations are performed on the basis of the testing arrangement $T_{6}=T_{6}(10)$. The comparison indicates that $\mathcal{T}_{f}\left(\boldsymbol{x}^{\circ}, \beta^{\text {true }}, \gamma^{\text {true }}\right)$ provides a markedly better indication of the obstacle location and shape, especially for $k \ell=4,8$.

The influence of $\beta$ on $\mathcal{T}_{f}$ is examined further in Fig. 9 , where $\mathcal{T}_{f}\left(\boldsymbol{x}_{c}^{\text {true }}, \beta, \gamma^{\text {true }}\right)$ (evaluated at the centroid of the true obstacle) is plotted against $\beta$ for scatterer configurations $\mathrm{P}-\mathrm{F}, \mathrm{P}-\mathrm{H}, \mathrm{R}-\mathrm{F}, \mathrm{R}-\mathrm{H}$ and resonance-region wavenumbers $k \ell=1,2,4$. Without exception, the band-limited "optimal" values $\beta=\beta_{\text {opt }}$ where $\mathcal{T}_{f}\left(\boldsymbol{x}_{c}^{\text {true }} ; \beta, \gamma^{\text {true }}\right)$ takes the maximum negative value are consistent with the mass densities of respective true scatterers $\left(\mathrm{P}: \beta^{\text {true }}=2, \mathrm{R}: \beta^{\text {true }}=0\right)$.

For completeness, the effect of $\gamma$ on $\mathcal{T}_{f}$ is examined in terms of $\mathcal{T}_{f}\left(\boldsymbol{x}_{c}^{\text {true }}, \beta^{\text {true }}, \gamma\right)$, again evaluated at the centroid of the true scatterer. In this case, all diagrams are by default linear in terms of $\gamma^{2}$, see (23) or (35). Unfortunately, the synthetic results generated for a number of configurations indicate that the band-limited "optimal" value of $\gamma$ is not always consistent with the constitutive nature of the respective true obstacle.

The foregoing results, and in particular those in Fig. 9, lend themselves to the idea of computing the "optimal" $\mathcal{T}_{f}$-distribution, obtained via point-wise minimization of $\mathcal{T}_{f}\left(\boldsymbol{x}^{\circ}, \beta, \gamma\right)$ with respect to $\beta$ so that

$$
\mathcal{T}_{f, \text { opt }}\left(\boldsymbol{x}^{\circ}\right) \equiv \mathcal{T}_{f}\left(\boldsymbol{x}^{\circ}, \beta_{\mathrm{opt}}\left(\boldsymbol{x}^{\circ}\right), \gamma^{\text {true }}\right),
$$

with the search interval for $\beta_{\mathrm{opt}}$ taken here as $0 \leq \beta \leq 4$. In this context, the key question is whether the "optimized" distribution of $\beta_{\mathrm{opt}}\left(\boldsymbol{x}^{\circ}\right)$ thus obtained is consistent with the true scatterer configuration. To facilitate the graphical interpretation, a thresholded variant of $\mathcal{T}_{f \text {,opt }}\left(\boldsymbol{x}^{\circ}\right)$ is introduced according to

$$
\hat{\mathcal{T}}_{f, \text { opt }}\left(\boldsymbol{x}^{\circ}\right)=\mathcal{T}_{f, \text { opt }}\left(\boldsymbol{x}^{\circ}\right)\left(\mathcal{T}_{f, \text { opt }} \leq C \mathcal{T}_{f, \text { opt }}^{\text {min }}\right), \quad \hat{\mathcal{T}}_{f, \text { opt }}\left(\boldsymbol{x}^{\circ}\right)=0\left(\mathcal{T}_{f, \text { opt }}>C \mathcal{T}_{f, \text { opt }}^{\text {min }}\right)
$$

where $\mathcal{T}_{f \text {, opt }}^{\text {min }}=\min _{\boldsymbol{x}^{\mathrm{o}} \in \mathcal{G}} \mathcal{T}_{\text {f,opt }}\left(\boldsymbol{x}^{\circ}\right)$ and $C=0.2+(k \ell)^{-1}$. In this way, an approximate image of the obstacle is formed through an assembly of sampling points where the 
optimal topological sensitivity $\mathcal{T}_{f, \text { opt }}^{\min }$ takes "sufficiently" high negative values relative to the global minimum, $\mathcal{T}_{f, \mathrm{opt}}^{\min }<0$. The featured choice of a frequency-dependent threshold $C$ is established on an ad-hoc basis, and reflects the fact that the $\mathcal{I}_{f}$-distributions are found to be more "smeared" at lower frequencies (see Figs. 3-6). On the basis of (38), a corresponding (thresholded) distribution of the optimal $\beta$-parameter is taken as

$$
\hat{\beta}_{\mathrm{opt}}\left(\boldsymbol{x}^{\circ}\right)=0\left(\hat{\mathcal{T}}_{f, \mathrm{opt}}\left(\boldsymbol{x}^{\circ}\right)<0\right), \quad \hat{\beta}_{\mathrm{opt}}\left(\boldsymbol{x}^{\circ}\right)=1\left(\hat{\mathcal{T}}_{f, \mathrm{opt}}\left(\boldsymbol{x}^{\circ}\right)=0\right) .
$$

The distributions of $\hat{\mathcal{T}}_{\text {,opt }}$ and $\hat{\beta}_{\text {opt }}$, obtained for the single-scatterer configurations $\mathrm{P}-\mathrm{F}$ and $\mathrm{R}-\mathrm{F}$, are shown in Figs. 10 and 11 for $k \ell=2,4,8$. For the resonance-region wavenumbers $(k \ell=2,4)$, the distributions of $\hat{\mathcal{F}}_{\text {,opt }}$ reasonably approximate the support of respective scatterers. The corresponding $\hat{\beta}_{\mathrm{opt}}$-distributions likewise take values that are consistent with the qualitative nature of the respective "true" obstacles. The results for the "intermediate" wavenumber $k \ell=8$, on the other hand, are mixed and are not deemed as effective as those obtained for the resonance region. The distributions of $\hat{\mathcal{T}}_{\text {fopt }}$ and $\hat{\beta}_{\text {opt }}$ obtained for the dual-scatterer configurations PP-H, PR-H and RR-H, shown in Figs. 12-14, lead to the same conclusion. In particular, the $k \ell=2,4$ distributions of $\hat{\beta}_{\text {opt }}$ obtained for configuration $\mathrm{PR}-\mathrm{H}$, which features one soft and one rigid obstacle, point to the correct nature of each scatterer.

For completeness, the level surfaces of $\mathcal{T}_{f, \text { opt }}\left(\boldsymbol{x}^{\circ}\right)$ defined by $\mathcal{T}_{f, \text { opt }}=C \mathcal{T}_{f \text {,opt }}^{\text {min }}$ where $C=0.2+(k \ell)^{-1}$ are computed over the entire 3D sampling grid $\mathcal{G}$ and graphically shown in Fig. 15 for $k \ell=2,8$ and scatterer configurations $\mathrm{PP}-\mathrm{H}, \mathrm{PR}-\mathrm{H}$ and $\mathrm{RR}-\mathrm{H}$. These three-dimensional plots in particular demonstrate that the values of topological sensitivity deemed significant (i.e. lower than the threshold value $C \mathcal{T}_{f, \mathrm{opt}}^{\min }$ ) do not occur outside a neighborhood of the scatterers, an observation which is found to hold for all configurations examined in this study.

\section{Conclusions}

In this paper, the concept of topological sensitivity is generalized to permit nucleation of penetrable obstacles as it pertains to the three-dimensional Helmholtz equation and inverse scattering problems in acoustics. In the context of arbitrarily-shaped inclusions, the featured formula is shown to consist of a dipole and a monopole term, related respectively to the density and compressibility contrast between the nucleating obstacle and a background medium. Explicit formulas are given for special cases when the nucleating inclusion takes spherical or ellipsoidal shape. The introduction of an adjoint solution further permits to consider nucleation of arbitrarily-shaped inclusions in an infinite, semi-infinite or finite background medium. To highlight the utility of proposed developments in dealing with inverse scattering problems, a set of numerical results is included wherein hidden obstacles are exposed through regions where (closedform) topological sensitivity attains negative values. On varying the mass density of a nucleating obstacle, it is also shown that the proposed methodology can be used in conjunction with long wavelengths (the so-called resonance region) for both geometric 
and material identification.

\section{Acknowledgments}

The support provided by the National Science Foundation through grants CMS-0324348 and INT-0340590 to B. Guzina and the National Center for Scientific Research, France's Ministry of Research, through award No. 17151 to M. Bonnet is gratefully acknowledged. The authors are also thankful to the University of Minnesota Supercomputing Institute for the computing resources made available during the course of this investigation.

\section{References}

[1] H. Ammari, E. Iakovleva, and S. Moskow. Recovery of small inhomogeneities from the scattering amplitude at a fixed frequency. SIAM J. Math. Anal., 34:882-90, 2003.

[2] H. Ammari and H. Kang. Reconstruction of the small inhomogeneities from boundary measurements. Springer, Berlin, New York, 2004.

[3] H. Ammari and A. Khelifi. Electromagnetic scattering by small dielectric inhomogeneities. J. Math. Pures Appl., 82:749-842, 2003.

[4] H. Ammari, S. Moskow, and M. Vogelius. Boundary integral formulae for the reconstruction of electric and electromagnetic inhomogeneities of small volume. ESAIM Control Optim. Calc. Var., 9:49-66, 2003.

[5] M. Bonnet. BIE and material differentiation applied to the formulation of obstacle inverse problems. Eng. Anal. with Bound. Elem., 15:121-136, 1995.

[6] M Bonnet. Boundary integral equations methods for solids and fluids. Wiley, 1999.

[7] M. Bonnet. Small-obstacle expansion in 3-d inverse scattering. In D. Lesnic, editor, Proceedings of the Fifth International Conference on Inverse Problems in Engineering: Theory and Practice (http://www.me.ua.edu/inverse/5icipe/), 2005.

[8] M. Bonnet and B. B. Guzina. Topological derivative for the inverse scattering of elastic waves. Int. J. Num. Meth. Eng., 57:161-179, 2004.

[9] J. Céa, S. Garreau, P. Guillaume, and M. Masmoudi. The shape and topological optimization connection. Comp. Meth. in Appl. Mech. Eng., 188:703-726, 2001.

[10] D. Colton, J. Coyle, and P. Monk. Recent developments in inverse acoustic scattering theory. SIAM Review, 42:369-414, 2000.

[11] D. Colton and A. Kirsch. A simple method for solving inverse scattering problems in the resonance region. Inverse Problems, 12:383-393, 1996.

[12] D. Colton and R. Kress. Inverse acoustic and electromagnetic scattering teory. Springer, Berlin, 1992.

[13] D. Colton and P. Monk. Numerical solution of an inverse scattering problem for acoustic waves. IMA J. Appl. Math., 49:163-184, 1992. 
[14] G. Dassios and P. Kleinman. Low frequency scattering. Oxford University Press, 2000 .

[15] C. Farhat, R. Tezaur, and R. Djellouli. On the solution of three-dimensional inverse obstacle acoustic scattering problems by a regularized newton method. Inverse Problems, 18:1229-1246, 2002.

[16] M. Fatemi and J. F. Greenleaf. Probing the dynamics of tissue at low frequencies with the radiation force of ultrasound. Phys. Med. Biol., 45:1449-1-464, 1998.

[17] G. R. Feijoo. A new method in inverse scattering based on the topological derivative. Inverse Problems, 20:1819-1840, 2004.

[18] R. Gallego and G. Rus. Identification of cracks and cavities using the topological sensitivity boundary integral equation. Comp. Mech., 33:154-163, 2004.

[19] S. Garreau, P. Guillaume, and M. Masmoudi. The topological asymptotic for PDE systems: the elasticity case. SIAM J. Control Optim., 39:1756-1778, 2001.

[20] B. B. Guzina and M. Bonnet. Topological derivative for the inverse scattering of elastic waves. Quart. J. Mech. Appl. Math., 57:161-179, 2004.

[21] B. B. Guzina and I. Chikichev. From imaging to material identification: a generalized concept of topological sensitivity. In Proceedings, ECCOMAS 2004 Congress, Jyvaskyla, Finland, 2004.

[22] B. B. Guzina, S. F. Nintcheu, and M. Bonnet. On the stress-wave imaging of cavities in a semi-infinite solid. Int. J. Solids Struct., 40:1505-1523, 2003.

[23] E. W. Hobson. The theory of spherical and ellipsoidal harmonics. Cambridge Univ. Press, London, U.K, 1931.

[24] A. Litman, D. Lesselier, and F. Santosa. Reconstruction of a two-dimensional binary obstacle by controlled evolution of a level-set. Inverse Problems, 14:685706, 1998.

[25] Z. Michalewicz and D. B. Fogel. How to solve it: modern heuristics. Springer Verlag, 2004.

[26] R. Pike and P. Sabatier, editors. Scattering: scattering and inverse scattering in pure and applied science, volume 1 \& 2. Academic Press, San Diego, 2002.

[27] J. Pommier and B. Samet. The topological asymptotic for the Helmholtz equation with Dirichlet condition on the boundary of an arbitrarily shaped hole. SIAM J. Control Optim., 43:899-921, 2005.

[28] R. Potthast. A survey on sampling and probe methods for inverse problems. Inverse Problems, 22:R1-R47, 2006.

[29] Lord Rayleigh. On the incidence of aerial and electric waves upon small obstacles in the form of ellipsoids or elliptic cylinders and on the passage of electric waves through a circular aperture in a conducting screen. Phil. Magazine, XLIV:28-52, 1897.

[30] B. Samet, S. Amstutz, and M. Masmoudi. The topological asymptotic for the Helmholtz equation. SIAM J. Control Optim., 42:1523-1544, 2004. 
[31] A. P. Sarvazyan, O. P. Rudenko, S. D. Swanson, J. B. Fowlkes, and S. Y. Emelyanov. Shear wave elasticity imaging: a new ultrasonic technology of medical diagnostics. Phys. Med. Biol., 24:1419-1435, 1998.

[32] J. Sokolowski and A. Zochowski. On the topological derivative in shape optimization. SIAM J. Control Optim., 37:1251-1272, 1999.

[33] A. Tarantola. Inverse problem theory and methods for model parameter estimation. SIAM, 2005.

[34] M. S. Vogelius and D. Volkov. Asymptotic formulas for perturbations in the electromagnetic fields due to the presence of inhomogeneities of small diameter. M2AN Math. Model. Num. Anal., 34:723-748, 2000.

[35] D. Volkov. Numerical methods for locating small dielectric inhomogeneities. Wave Motion, 38:189-206, 2003.
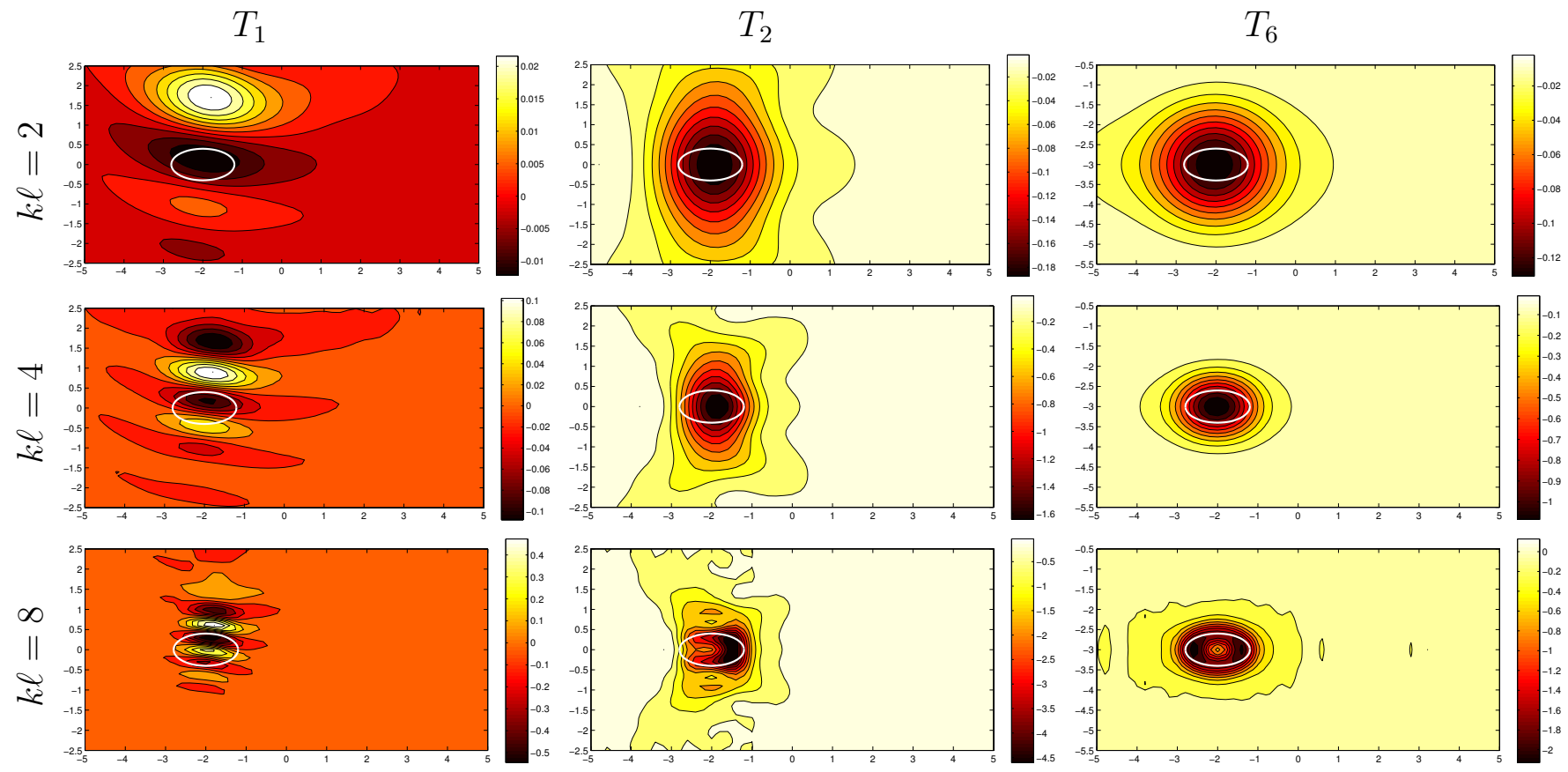

Figure 3. Influence of testing aperture: distribution of $\left(\ell \rho^{-2} c^{-4}\right) \mathcal{T}_{f}\left(\boldsymbol{x}^{\mathrm{o}}, \beta^{\text {true }}, \gamma^{\text {true }}\right)$ in the vertical plane $\xi_{2}=0.5 \ell$ for the scatterer configuration $\mathrm{P}-\mathrm{F}$. 

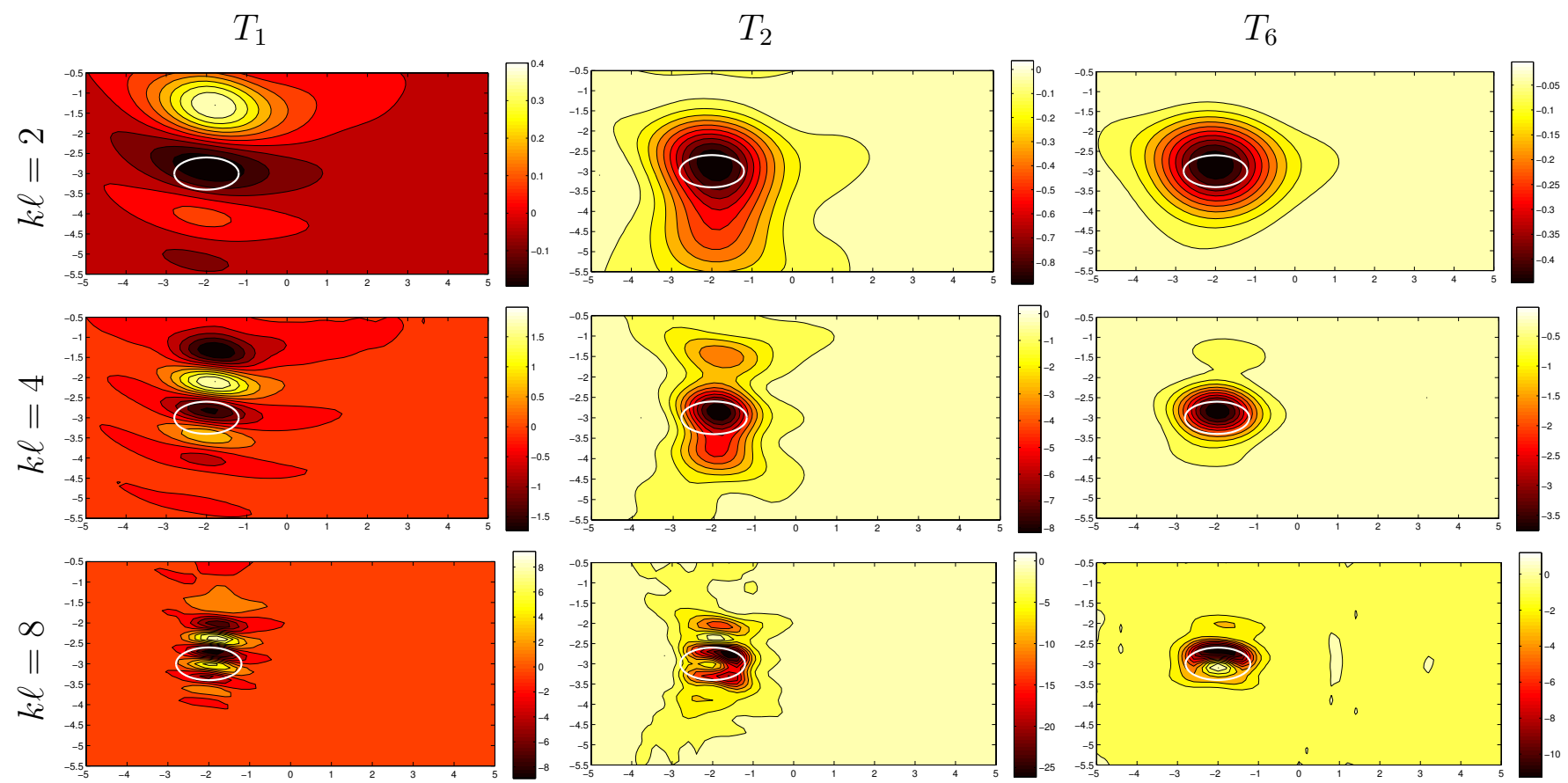

Figure 4. Influence of testing aperture: distribution of $\left(\ell \rho^{-2} c^{-4}\right) \mathcal{T}_{f}\left(\boldsymbol{x}^{\mathrm{o}}, \beta^{\text {true }}, \gamma^{\text {true }}\right)$ in the vertical plane $\xi_{2}=0.5 \ell$ for the scatterer configuration $\mathrm{P}-\mathrm{H}$.
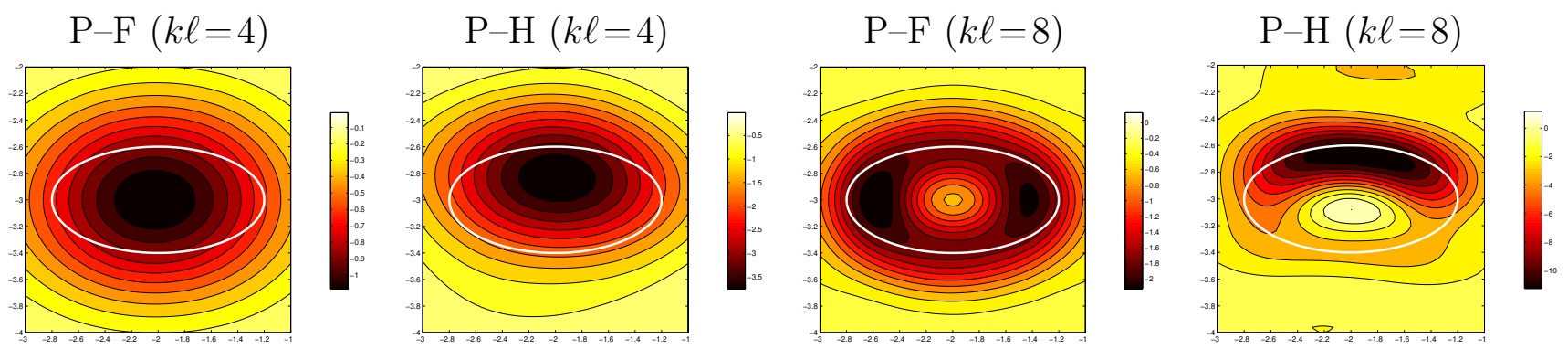

Figure 5. Near-obstacle detail of the selected graphs in Figures 3 and 4: full-aperture testing configuration $T_{6}$. 


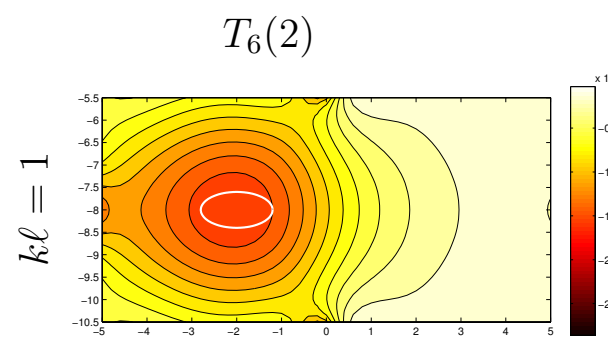

$T_{6}(5)$
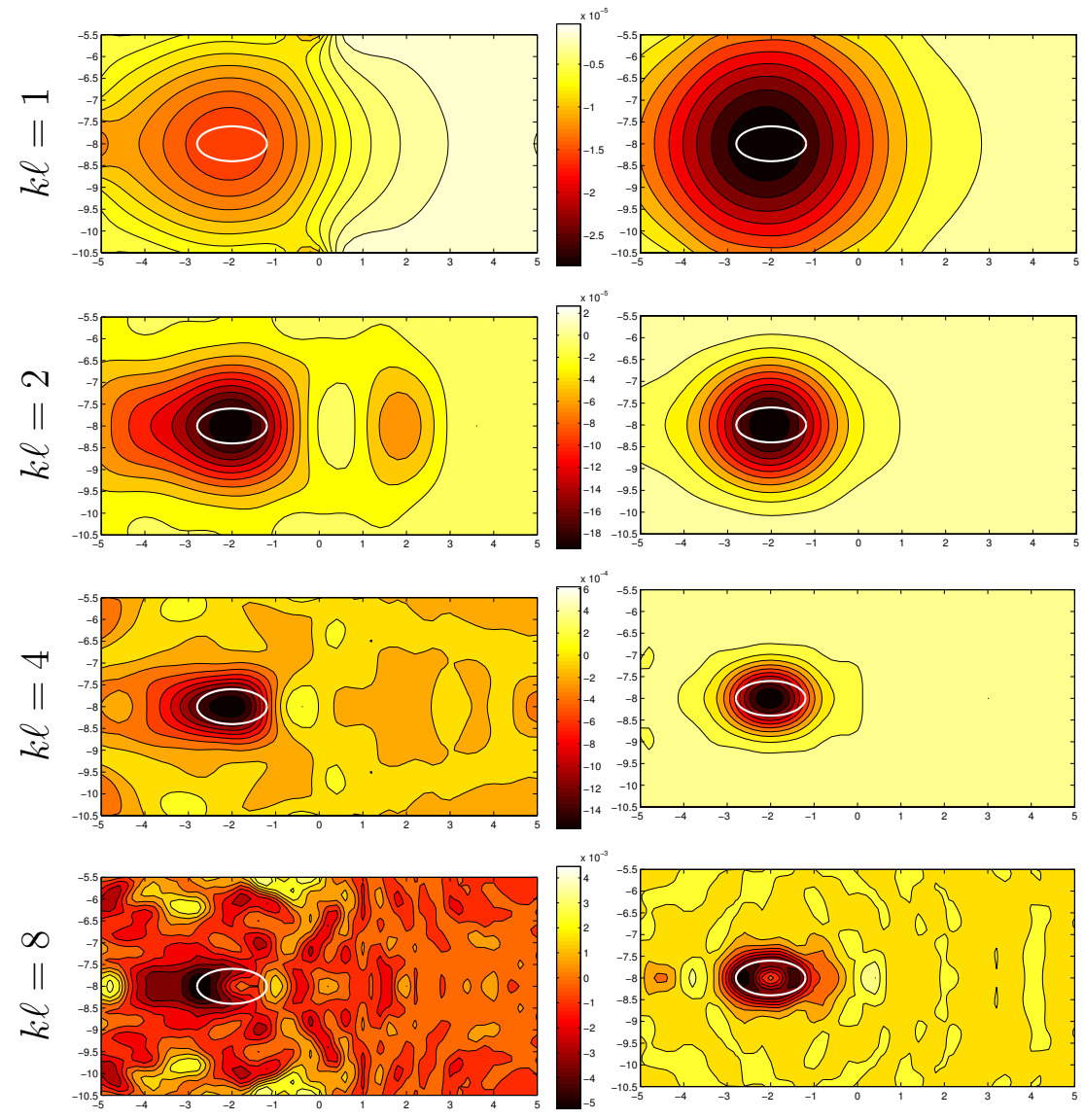
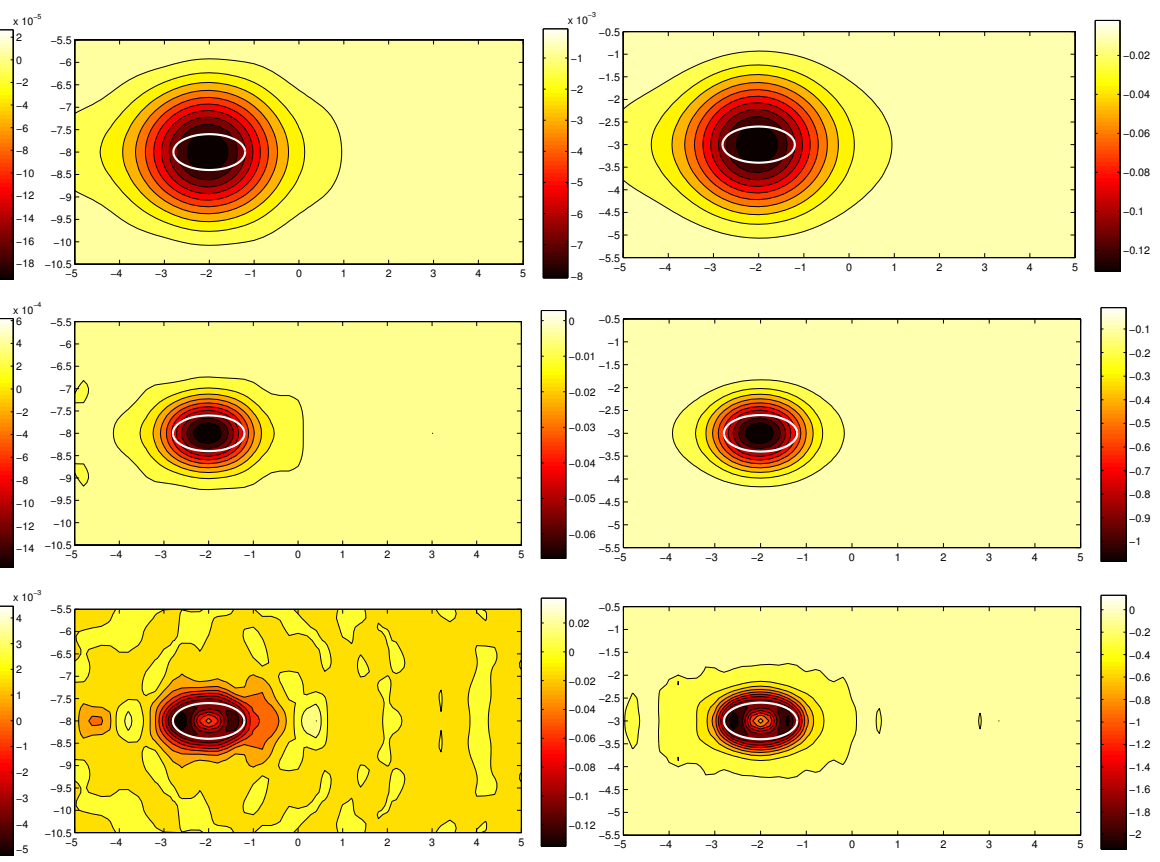

Figure 6. Influence of testing grid density ( $\mathrm{P}-\mathrm{H}$ configuration): distribution of $\left(\ell \rho^{-2} c^{-4}\right) \mathcal{T}_{f}\left(\boldsymbol{x}^{\mathrm{o}}, \beta^{\text {true }}, \gamma^{\text {true }}\right)$ in the vertical plane $\xi_{2}=0.5 \ell$, calculated for the testing arrangements $T_{6}(2), T_{6}(5)$ and $T_{6}(10)=T_{6}$. 


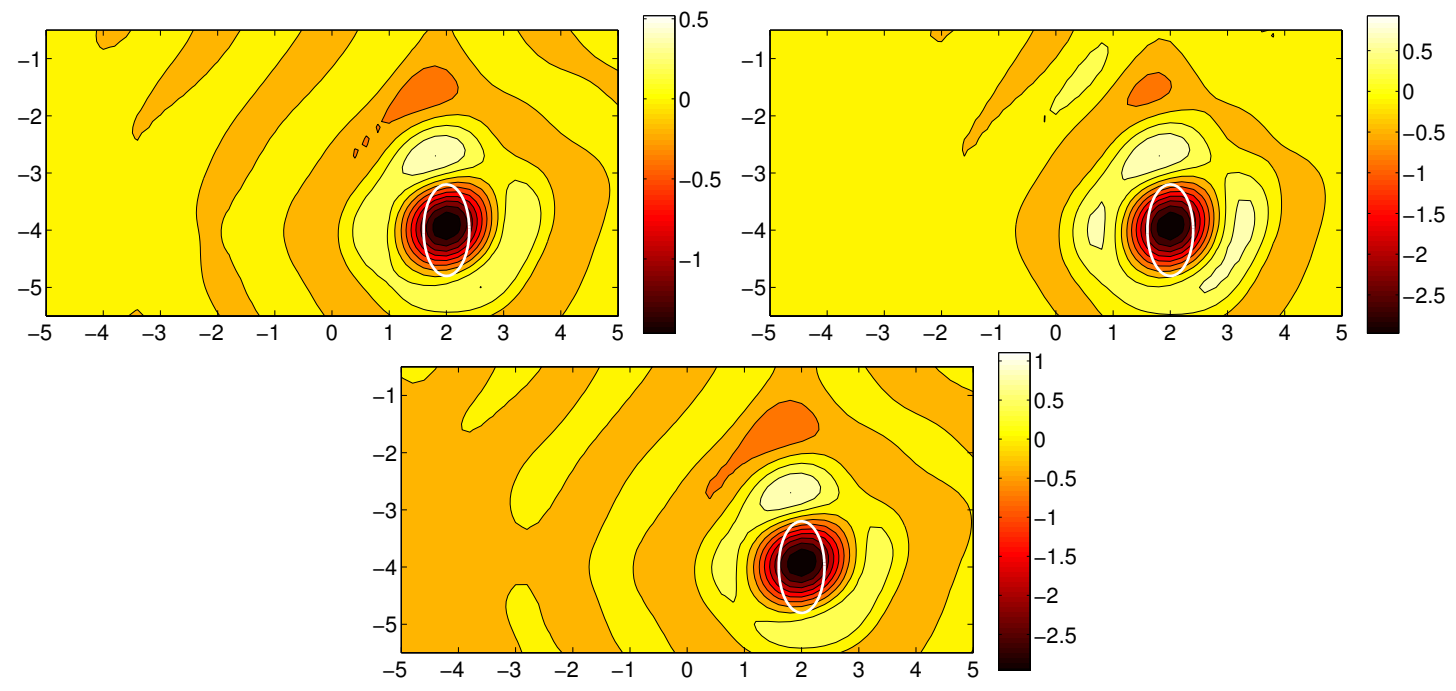

Figure 7. Influence of the shape of trial obstacle ( $\mathrm{R}-\mathrm{H}$ configuration): distribution of $\left(\ell \rho^{-2} c^{-4}\right) \mathcal{T}_{f}\left(\boldsymbol{x}^{\circ}, \beta^{\text {true }}, \gamma^{\text {true }}\right)$ in the vertical plane $\xi_{2}=0.5 \ell$ for $k \ell=2$ assuming $a_{1}=a_{2}=a_{3}=\epsilon$ (top left), $2 a_{1}=2 a_{2}=a_{3}=\epsilon$ (top right), and $a_{1}=2 a_{2}=2 a_{3}=\epsilon$ (bottom).
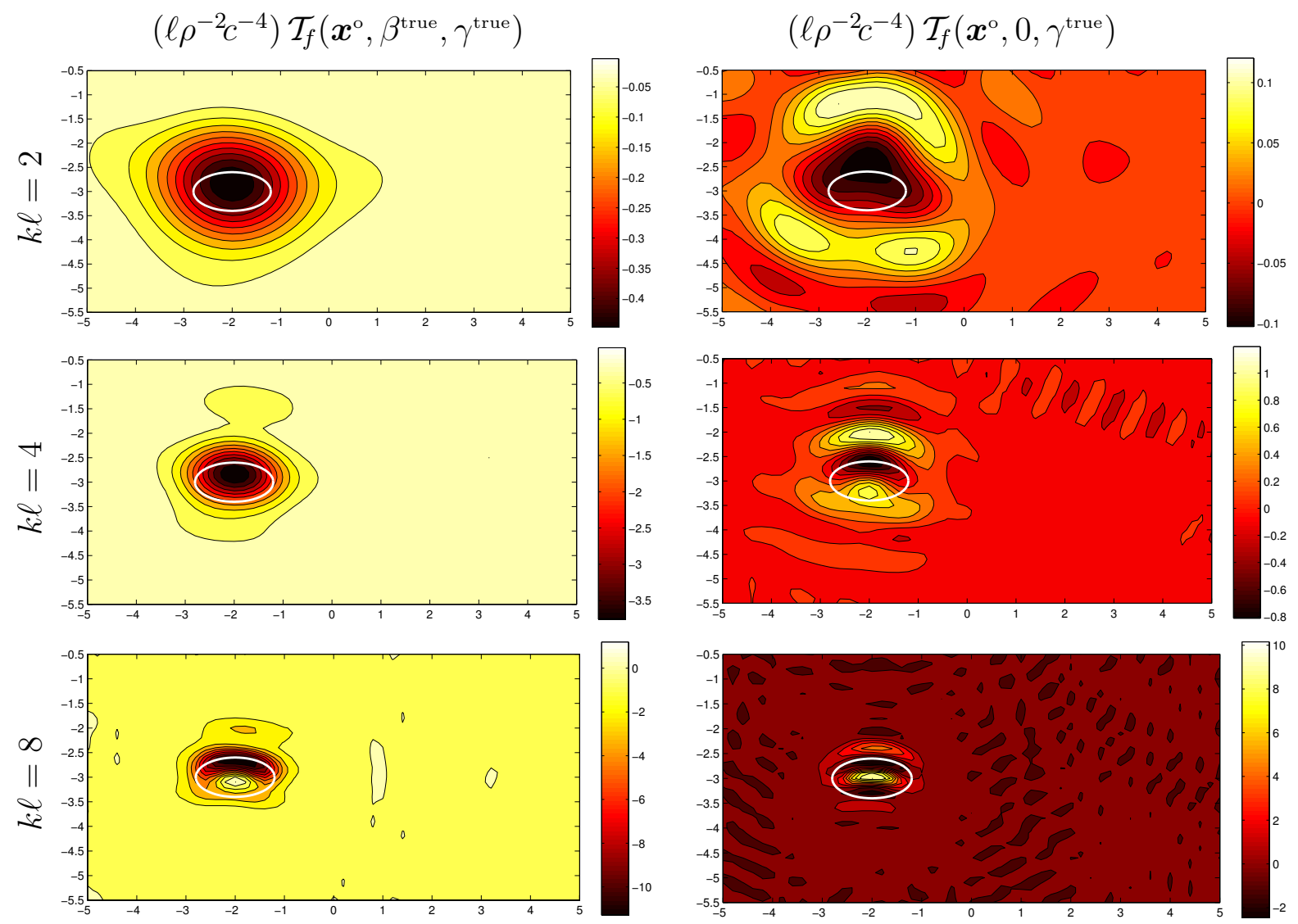

Figure 8. Influence of trial obstacle parameters ( $\mathrm{P}-\mathrm{H}$ configuration): distribution of $\mathcal{I}_{f}\left(\boldsymbol{x}^{\mathrm{o}}, \beta^{\text {true }}, \gamma^{\text {true }}\right)$ (left) and $\mathcal{T}_{f}\left(\boldsymbol{x}^{\circ}, 0, \gamma^{\text {true }}\right)$ (right) in the vertical plane $\xi_{2}=0.5 \ell$. 
$\mathrm{P}-\mathrm{F}$

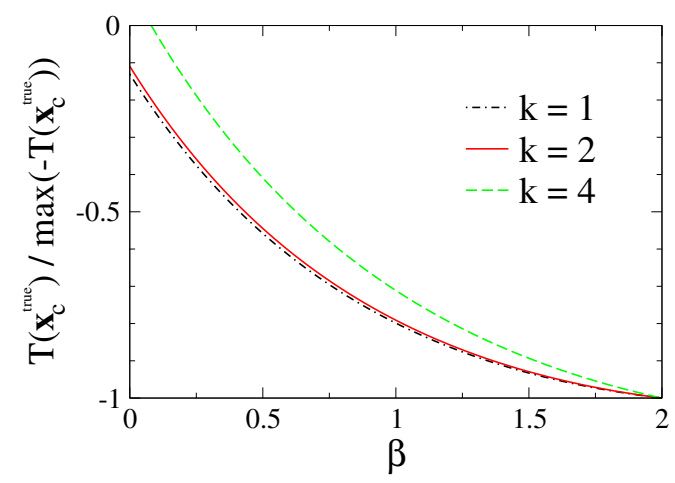

$\mathrm{R}-\mathrm{F}$

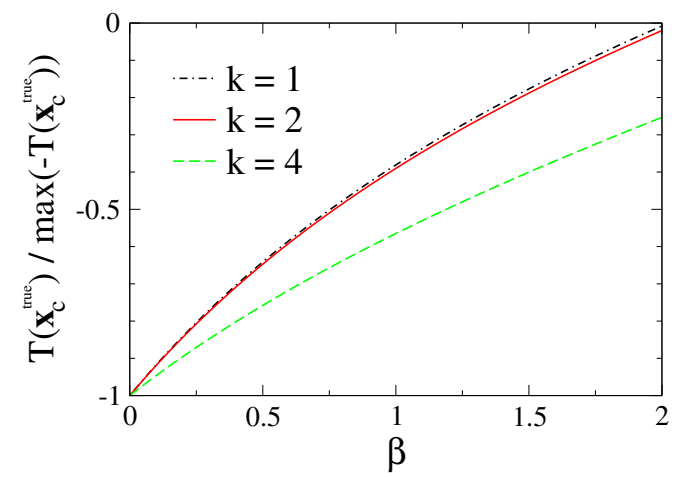

$\mathrm{P}-\mathrm{H}$

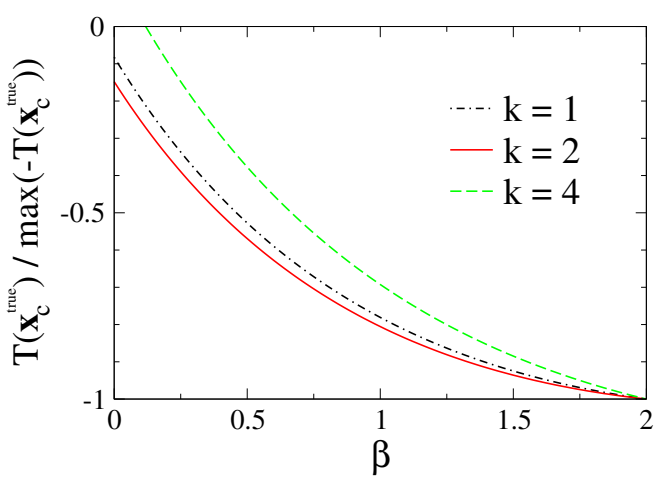

$\mathrm{R}-\mathrm{H}$

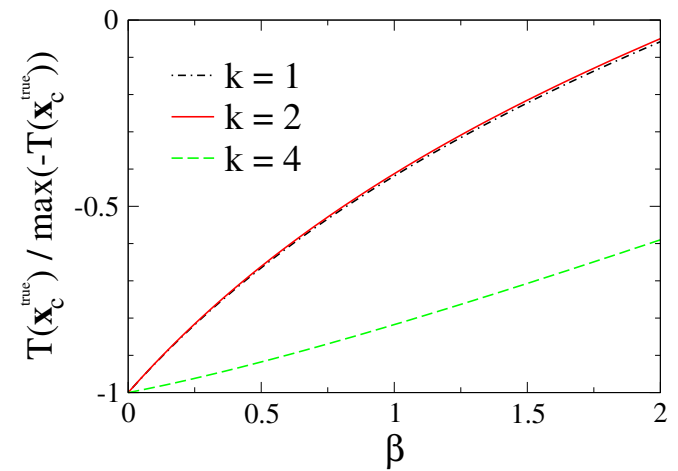

Figure 9. Variation of topological sensitivity $\mathcal{I}_{f}\left(\boldsymbol{x}_{c}^{\text {true }} ; \beta, \gamma^{\text {true }}\right)$ with $\beta$ at the centroid of true obstacle for the $\mathrm{P}-\mathrm{F}, \mathrm{P}-\mathrm{H}, \mathrm{R}-\mathrm{F}$ and $\mathrm{R}-\mathrm{H}$ configurations.
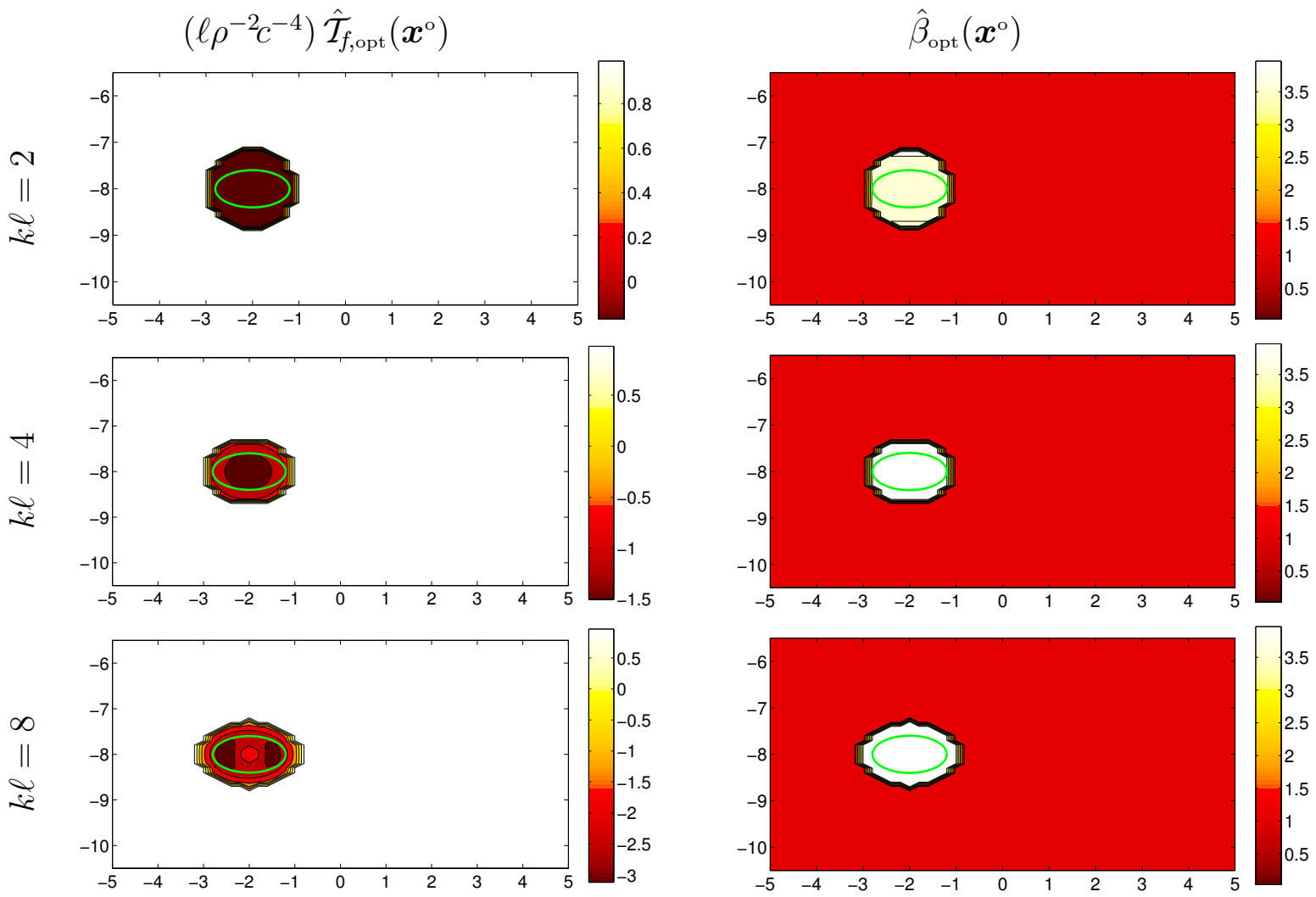

Figure 10. Distribution of $\hat{\mathcal{T}}_{\text {, opt }}\left(\boldsymbol{x}^{\circ}\right)$ and $\beta_{\mathrm{opt}}\left(\boldsymbol{x}^{\circ}\right)$ (P-F configuration). 

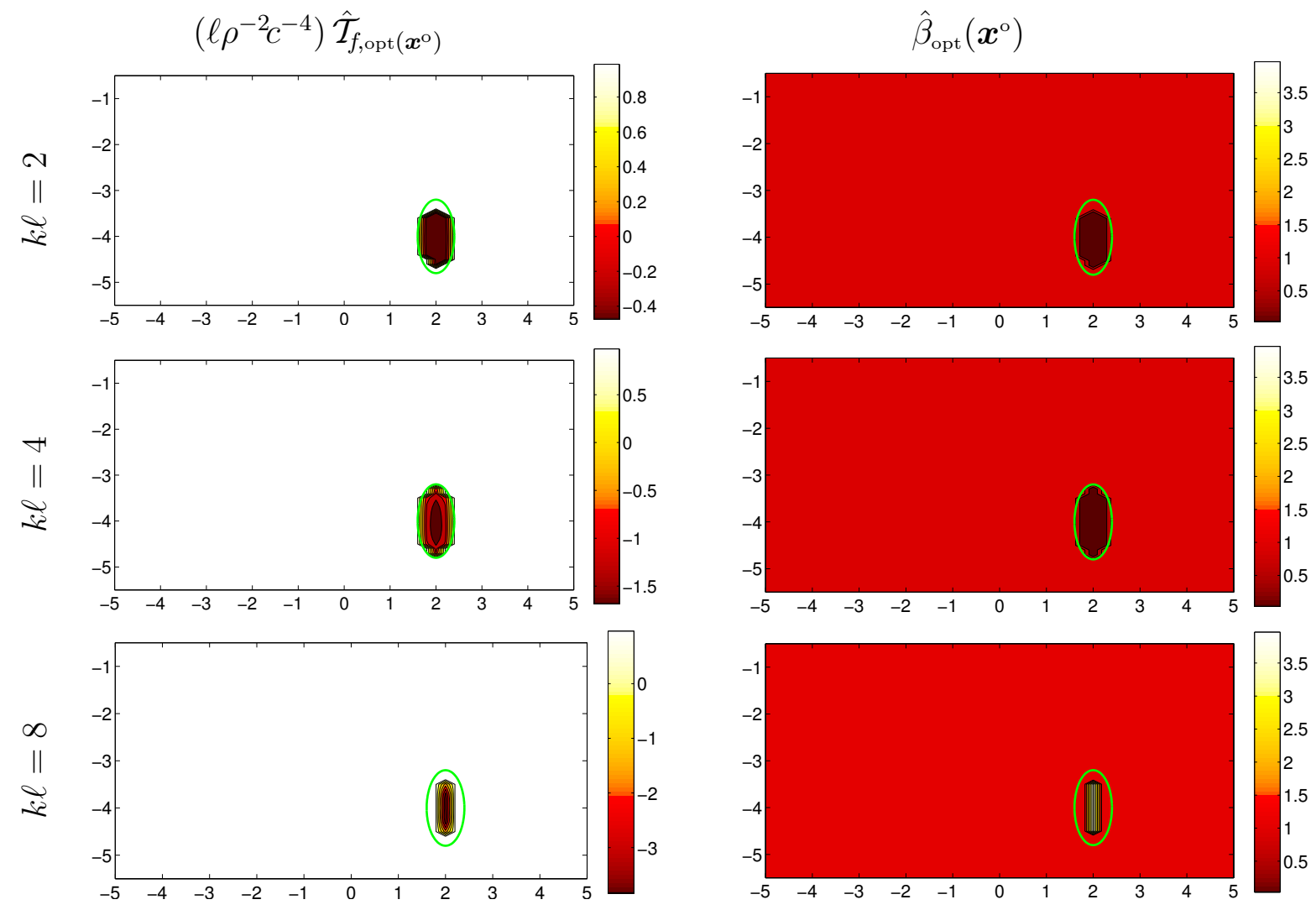

Figure 11. Distribution of $\hat{\mathcal{T}}_{f, \mathrm{opt}}\left(\boldsymbol{x}^{\circ}\right)$ and $\beta_{\mathrm{opt}}\left(\boldsymbol{x}^{\circ}\right)$ (R-F configuration).
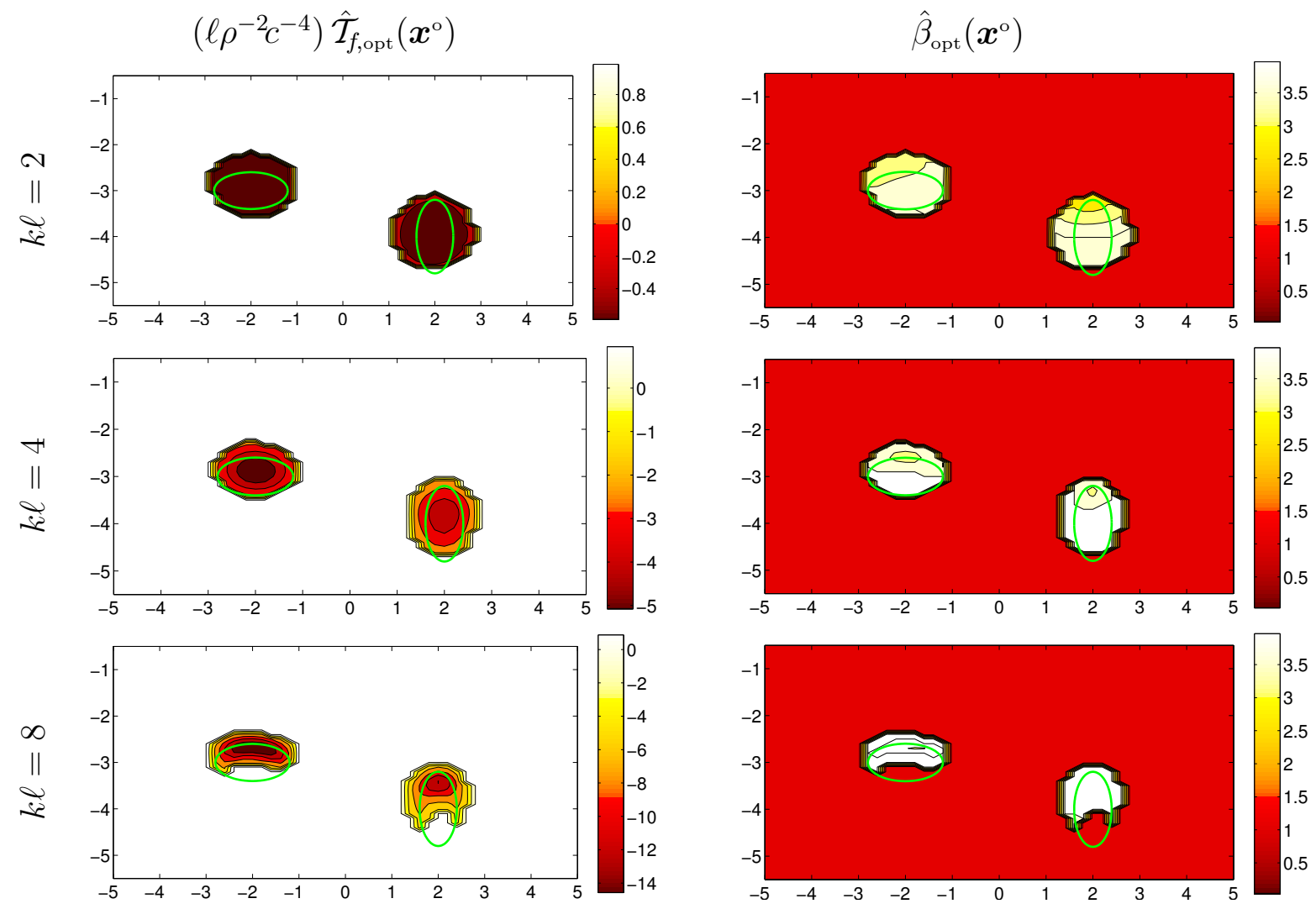

Figure 12. Distribution of $\hat{\mathcal{T}}_{f, \mathrm{opt}}\left(\boldsymbol{x}^{\circ}\right)$ and $\beta_{\mathrm{opt}}\left(\boldsymbol{x}^{\circ}\right)$ (PP-H configuration). 

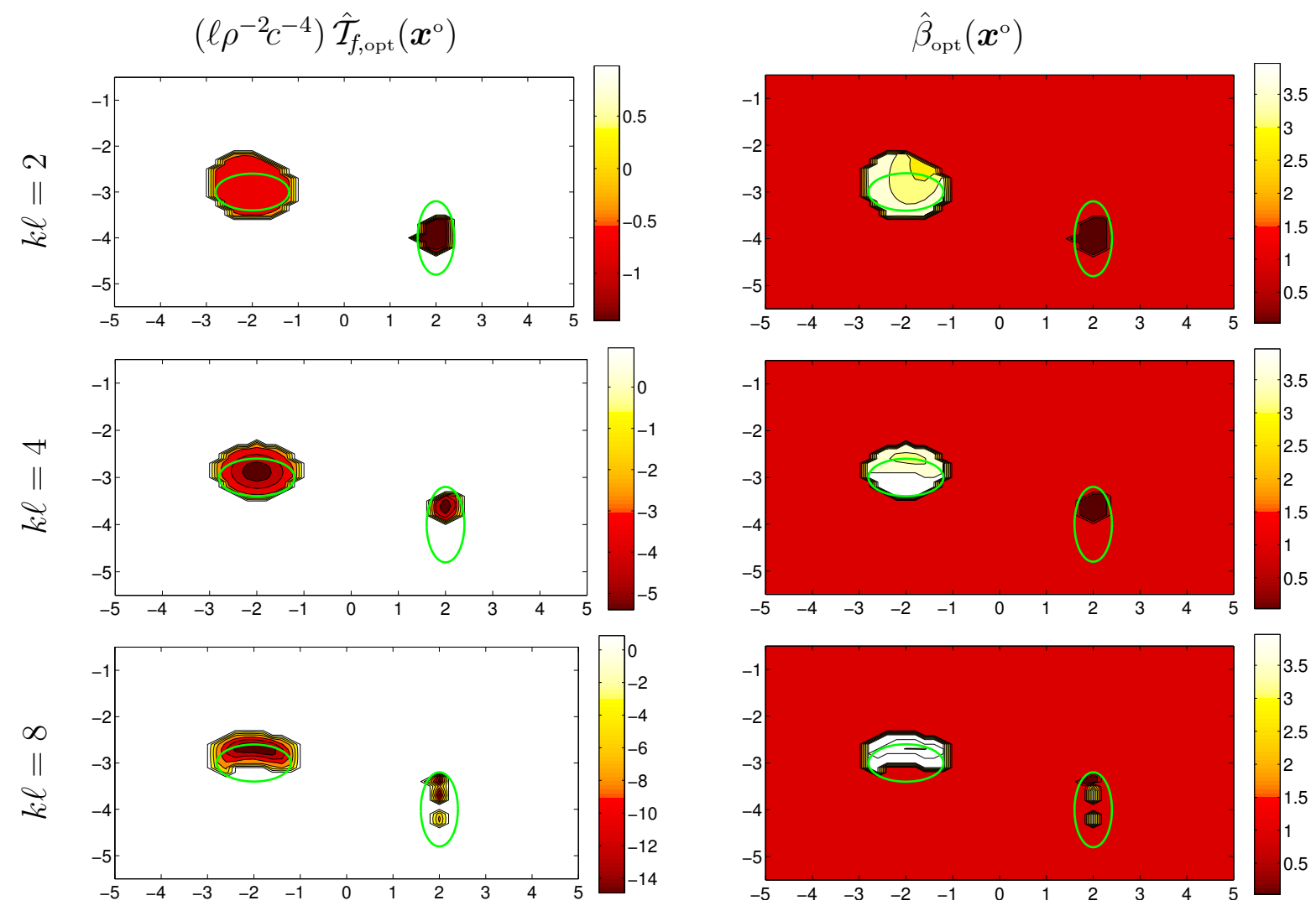

Figure 13. Distribution of $\hat{\mathcal{T}}_{\text {, opt }}\left(\boldsymbol{x}^{\circ}\right)$ and $\beta_{\mathrm{opt}}\left(\boldsymbol{x}^{\circ}\right)$ (PR-H configuration).
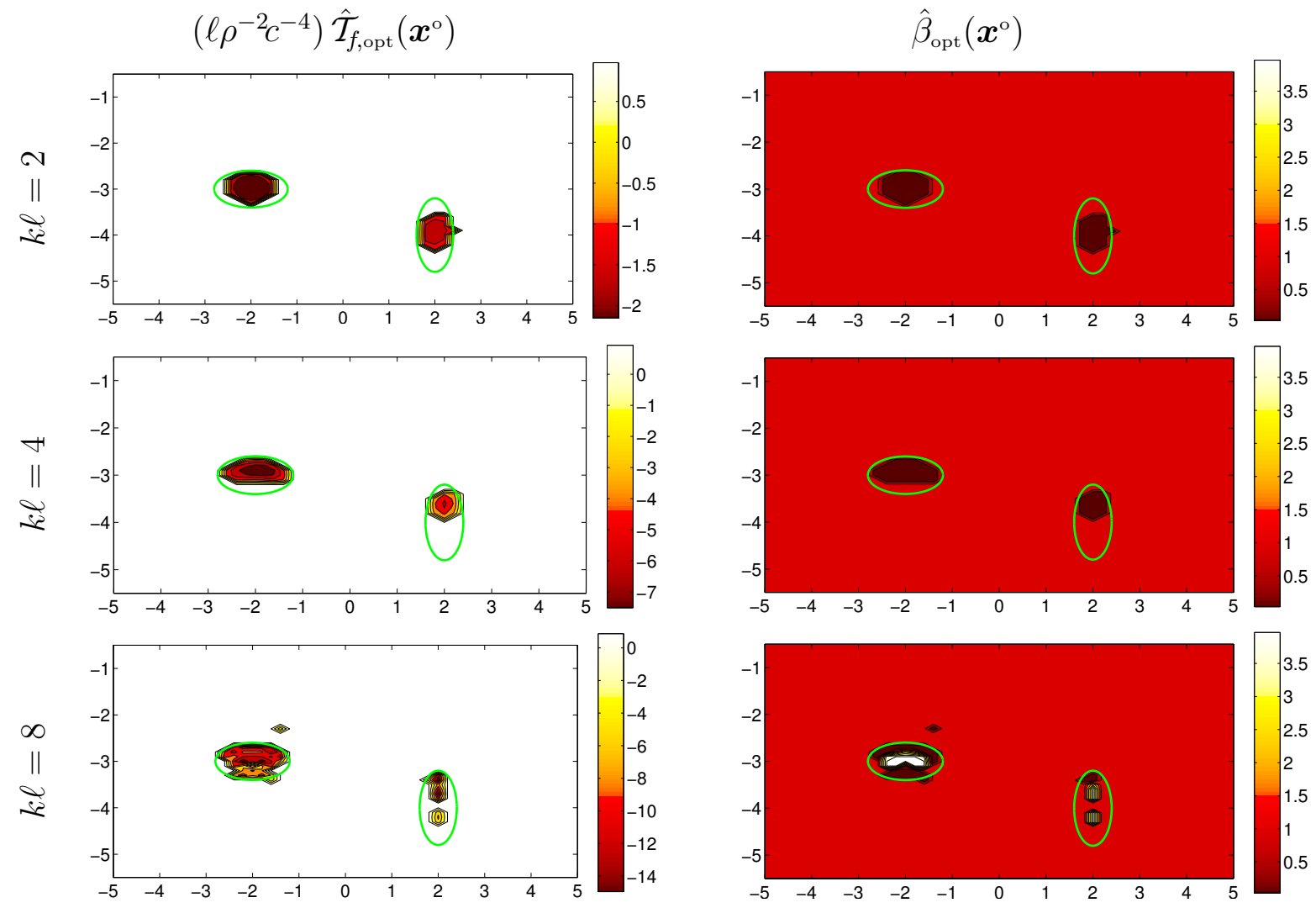

Figure 14. Distribution of $\hat{\mathcal{T}}_{\text {, opt }}\left(\boldsymbol{x}^{\circ}\right)$ and $\beta_{\text {opt }}\left(\boldsymbol{x}^{\circ}\right)$ (RR-H configuration). 

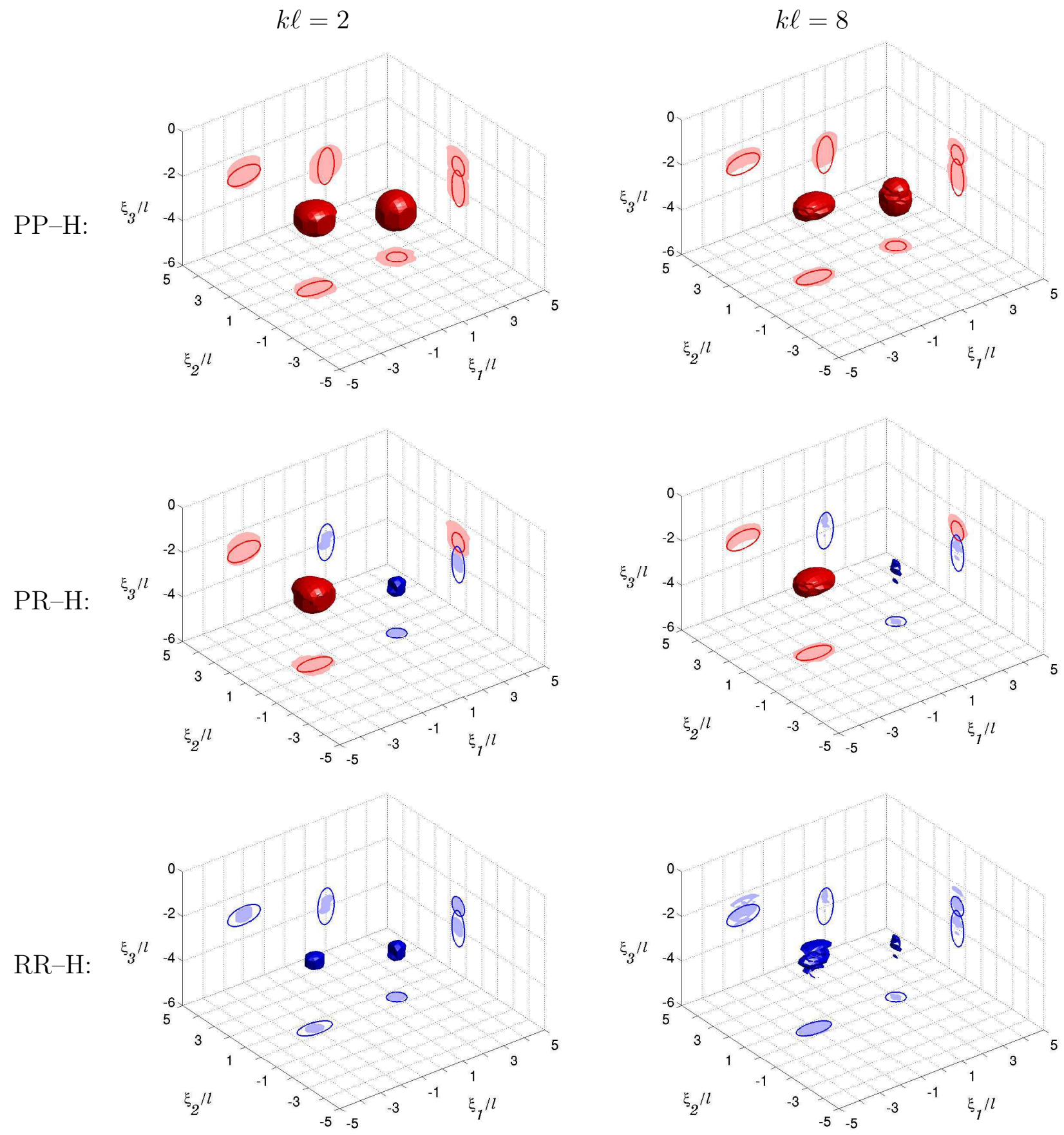

Figure 15. Level surfaces of $\mathcal{T}_{f \text {,opt }}\left(\boldsymbol{x}^{\circ}\right)$ defined by $\mathcal{T}_{f, \mathrm{opt}}=C(k \ell) \mathcal{T}_{f, \mathrm{opt}}^{\min }$, for $k \ell=2,8$ and scatterer configurations $\mathrm{PP}-\mathrm{H}, \mathrm{PR}-\mathrm{H}$ and $\mathrm{RR}-\mathrm{H}$. 